\title{
Supramolecular Thiophene-Based Materials: A Few Examples of the Interplay between Synthesis, Optoelectronic Properties and Applications
}

\author{
Francesca Di Maria*a (D) \\ Mattia Zangolia (i) \\ Giovanna Barbarella*a (D) \\ a ISOF, CNR Area della Ricerca di Bologna, Via P. Gobetti 101, 40129 Bologna, Italy \\ francesca.dimaria@isof.cnr.it; giovanna.barbarella@isof.cnr.it
}

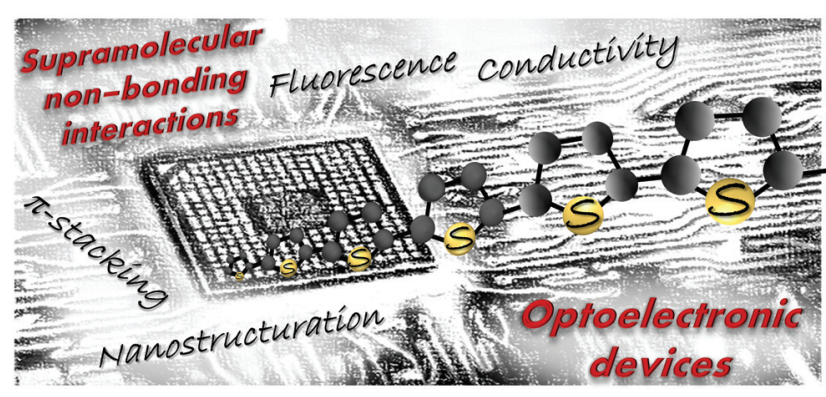

arouse wide interest for their easy synthesis and multiple tunable physicochemical and biological properties. Applications spreading from medicinal and pharmaceutical chemistry to materials chemistry, organic (bio)electronics and sensing have been reported. ${ }^{1}$ Several papers highlight the importance of elucidating the interplay between molecular structure, supramolecular interactions and crucial properties such as charge transport and light emission in order to optimize the possible applications. A remarkable characteristic of the thiophene ring - one of the most commonly found building blocks in semiconducting conjugated materials - is the possibility to be functionalized in different positions with different groups.This is one of the main factors at the origin of the great chemical diversity of thiophene derivatives. Scheme 1 illustrates the various positions where substituents can be grafted.

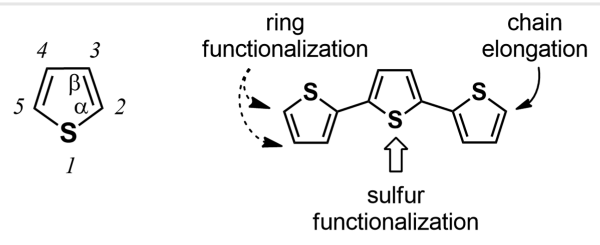

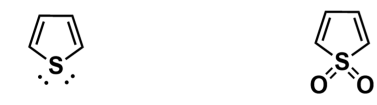
aromatic non-aromatic

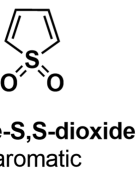

thiophene thiophene-S,S-dioxide
Scheme 1 Molecular structure and numbering scheme of thiophene, and functionalization positions.

\section{Introduction}

Despite the numerous investigations carried out over the last three decades, thiophene-based compounds continue to
The most studied functionalization type is that with substituents at positions $2-5$, in particular with alkyl chains. However, owing to its diffuse and very polarizable electrons, the sulfur atom may be hypervalent, i.e., it can accommodate more electrons than the eight ones associated 
Biosketches
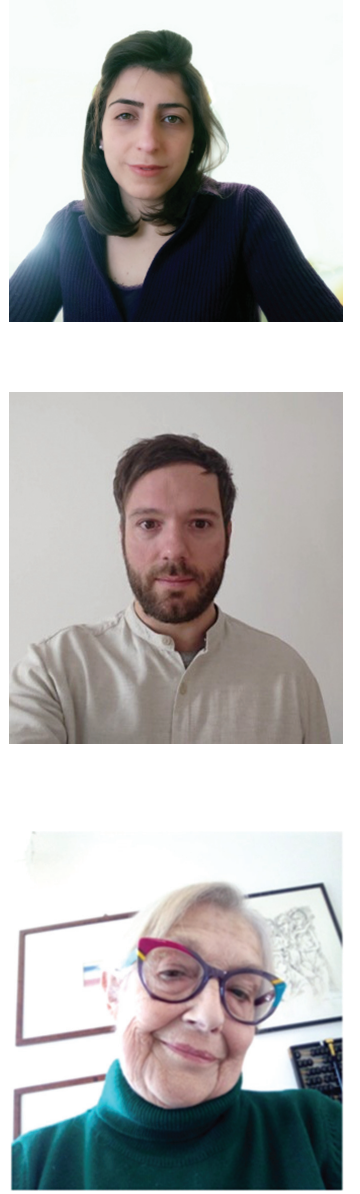

Giovanna Barbarella graduated at the University of Bologna (Italy) and received her PhD in 1974 from the University of Grenoble (France). Currently, she is an associate re-
Mattia Zangoli received his $\mathrm{PhD}$ in chemistry in 2018 from Bologna University. Then, he joined the $\mathrm{Di}$ Maria-Barbarella's research group as a post-doc researcher at the Institute
Photoreactivity (ISOF) of the Italian National Research Council (ISOFCNR). Her main research activity deals with the synthesis and characterization of advanced organic materials and their organization into supramolecular nano- and microstructures (0D and 1D) for optoelectronic and biological applications. of Organic Synthesis and Photoreactivity (ISOF) of the Italian National Research Council (CNR). He is currently a researcher of the ISOF-CNR. He is interested in the design and synthesis of oligo- and polythiophenes and their structure-property relationship. searcher at the Institute of Organic Synthesis and Photoreactivity (ISOF) of the Italian National Research Council. Her activity is focused on thiophene-based materials and their applications. with filled s and p shells and form a variety of groups with coordination number four and six. In materials chemistry only sulfur functionalization with oxygen has been explored so far (at position 1), giving rise to S,S-dioxides and S-oxides as illustrated in Scheme 1.

Whatever their nature and ring position is, the substituents determine the type of intra- and intermolecular interactions hence the supramolecular structure generated under given experimental conditions, the optoelectronic properties and the type of application in devices. A peculiarity of the thiophene ring is its 'plasticity', a concept defined in 1993 and derived from the observation that in oligothiophene (OT) single crystals the thiophene ring always displays an irregular pentagonal geometry. ${ }^{2}$ The plasticity of thiophene was defined as the capability of the ring to be deformed in order to relieve steric strain by means of small and progressive angle and bond changes while maintaining the planar geometry. Once again, this property was ascribed to the easily distorted clouds of electrons of the sulfur atom, which are at the origin of the great tendency of thiophene-based molecules to form aggregates through numerous different inter- and intramolecular non- bonding interactions with neighboring molecules. Because of the intrinsic plasticity of thiophene coupled to the low energy barriers to carbon-carbon inter-ring rotations, ${ }^{3}$ thiophenebased supramolecular architectures are difficult to predict only on the basis of the structure of the composing molecules. The most diverse ways of aggregation are possible, largely depending on the environment and the experimental conditions. Figure 1 shows some significant examples of how thiophene molecules can self-assemble/self-organize or even co-assemble with neighbouring molecules.

Figure $1 \mathrm{~A}$ shows the optical micrographs under polarized and unpolarized light of a melted drop of $\alpha$-sexithiophene, one of the most investigated thiophene oligomers for its supramolecular structures in relation to charge transport in field-effect transistors (T6, molecular structure in Scheme 2). ${ }^{4}$ Under polarized light, Maltese crosses, typical of liquidcrystalline nematic phases, are visible ${ }^{5}$ (see Supporting Information of ref. ${ }^{16}$ ). Figure $1 \mathrm{~B}$ shows the atomic force microscopy (AFM) image of thin films prepared by vacuum evaporation of powders of $\alpha$-quinquethiophene (T5, molecular structure in Scheme 2) displaying uniaxially aligned 
stripes and self-affine (fractal) morphology, i.e., the same type of hierarchical self-organization over several orders of magnitude in scale. ${ }^{6}$ Note that many natural and metallic surfaces display self-affinity over several scale orders. ${ }^{7}$ Figure $1 \mathrm{C}$ displays the AFM image of a microfiber formed inside live neuroblastoma cells ${ }^{8}$ by spontaneous co-assembly of the protein vimentin with the green fluorescent thiophene-based dye DTTO (3,5-dimethyl-2,3'-bis(phenyl)dithieno[3,2-b;2',3'-d]thiophene-4,4-dioxide; molecular structure in Scheme $3^{9}$ ). Being green fluorescent, DTTO confers green fluorescence to the microfiber, which consequently can be isolated from the cell and analyzed. Chernyatina et al. reported the calculated structure of the vimentin dimer ${ }^{10}$ which displays an astonishing similarity to the AFM image of the very peculiar shape of the microfiber.

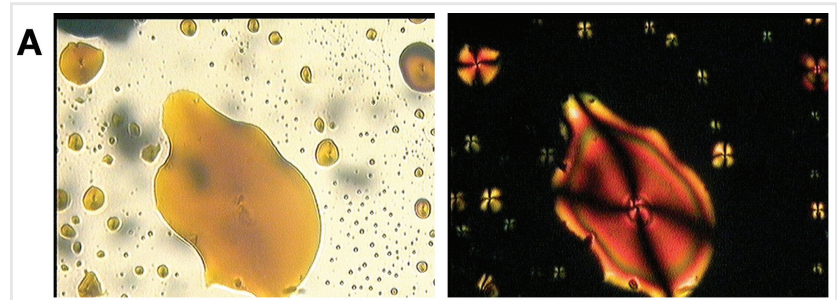

B

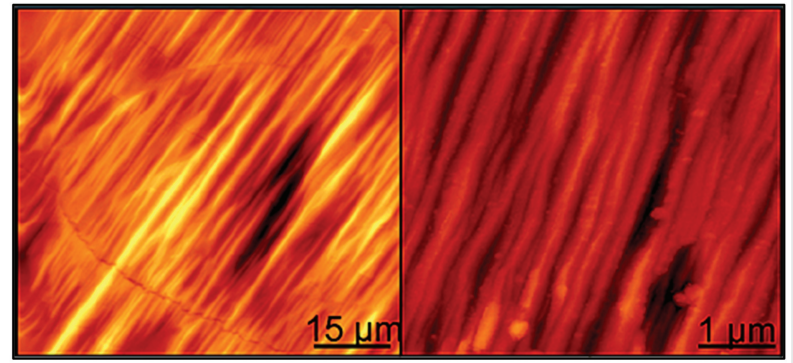

C

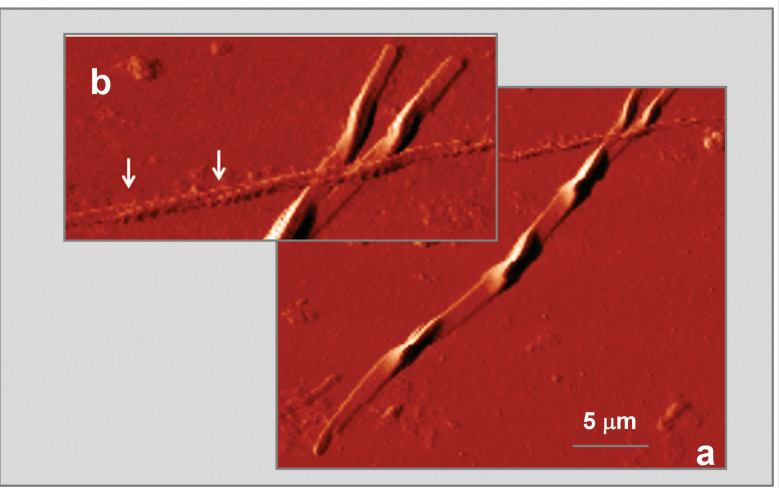

Figure 1 A) Optical micrographs under polarized (right) and unpolarized (left) light of a melted drop of compound T6. Reprinted with permission from Ref. 5 (SI). Copyright 2004 American Chemical Society. B) AFM image of $\alpha$-quinquethiophene melt-quenched on glass. Reprinted with permission from Ref. 6. Copyright 2003 American Chemical Society. C) AFM image of a microfiber formed inside live neuroblastoma cells by spontaneous co-assembly of the protein vimentin with the green fluorescent thiophene-based dye DTTO. Adapted with permission from Ref. 8. Copyright 2015 Royal Society of Chemistry.

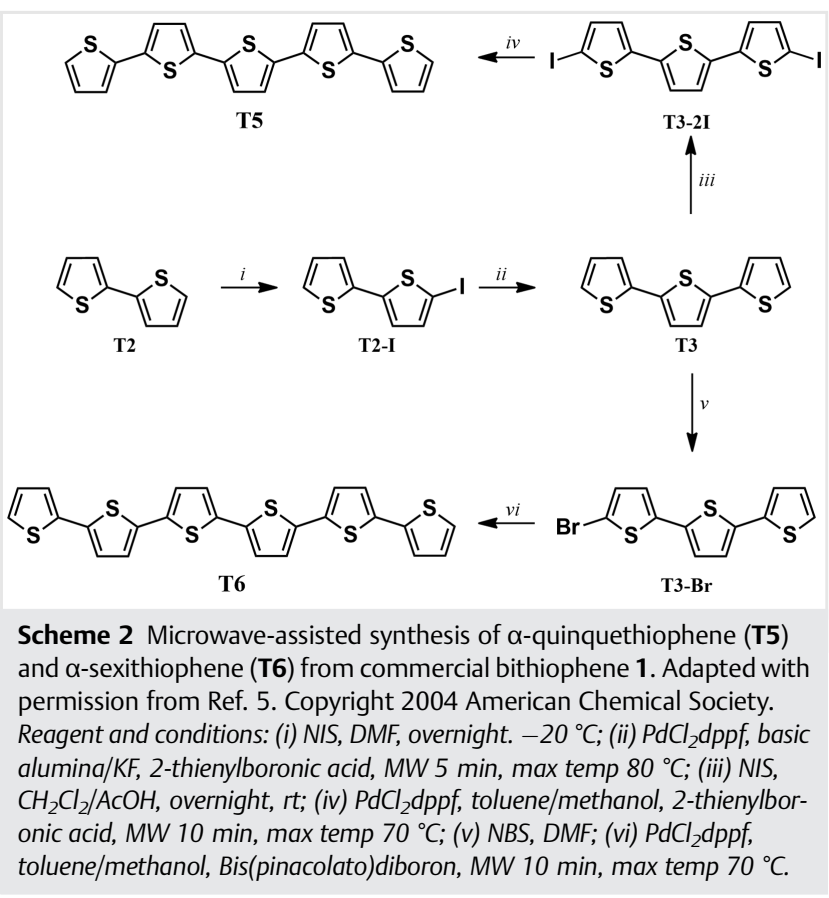

This suggests that there is a fractal growth of the coassembled supramolecular structure of the microfiber over a great number of orders of magnitude in scale. Scheme 2 shows the pattern for the synthesis of $\mathbf{T 5}$ and $\mathbf{T 6} \mathbf{6}^{5}$ generating the supramolecular structures in panels A and B of Figure 1, while Scheme 3 illustrates the synthesis of DTTO ${ }^{9}$ used in the coassembly of vimentin-DTTO bioconjugate shown in Figure $1 \mathrm{C}$.

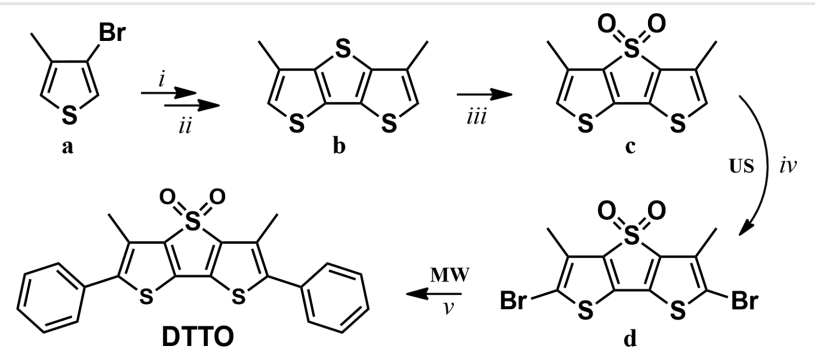

Scheme 3 Synthesis of 3,5-dimethyl-2,3'-bis(phenyl)dithieno[3,2b;2',3'-d]thiophene-4,4-dioxide (DTTO). Adapted with permission from Ref. 9a. Copyright 2011 American Chemical Society. Reagents and conditions: i) Bis(tri-n-butyltin) sulfide, $\mathrm{Pd}\left(\mathrm{PPh}_{3}\right)_{4}$., toluene, $130{ }^{\circ} \mathrm{C}, 90 \%$; ii) n-BuLi, $\mathrm{CuCl}$, ethyl ether, $0^{\circ} \mathrm{C}, 50 \%$; iii) 3-chloro-perbenzoic acid, $\mathrm{CH}_{2} \mathrm{Cl}_{2}$, $70 \%$; iv) $2 \mathrm{mmol}$ of NBS, $\mathrm{CH}_{3} \mathrm{COOH} / \mathrm{CH}_{2} \mathrm{Cl}_{2}$, US, 99\%; v) tributyl(phenyl) stannane $5 \%, P d\left(P P h_{3}\right)_{4}$, toluene, $M W, 80{ }^{\circ} \mathrm{C}, 95 \%$.

It is worth noting that the preparation of materials with reproducible optoelectronic features requires absence of any trace of byproducts or contaminants. Such a purity degree requires generally several purification steps or alternative ecofriendly synthetic patterns, as in the case of $\mathbf{T 5}$ and $\mathbf{T 6}$, which have also been prepared in the absence of solvent ${ }^{11}$ or in aqueous media using silica and chitosan-supported Pd catalysts. ${ }^{12}$ 


\section{Supramolecular Organization may Impart New Functions to the System}

When a supramolecular structure is formed under given experimental conditions, it may acquire new properties different from those pertaining to the building molecules and enable new applications. A few examples illustrating this point are shown below.

\subsection{White Organic Light Emitting Diode from a Single Compound}

The molecular structure of the compound in question, namely 3,5-dimethyl-2,6-bis(dimesitylboryl)-dithieno[3,2$\left.b: 2^{\prime}, 3^{\prime}-d\right]$ thiophene, is reported in Figure $2 \mathrm{~A}$.

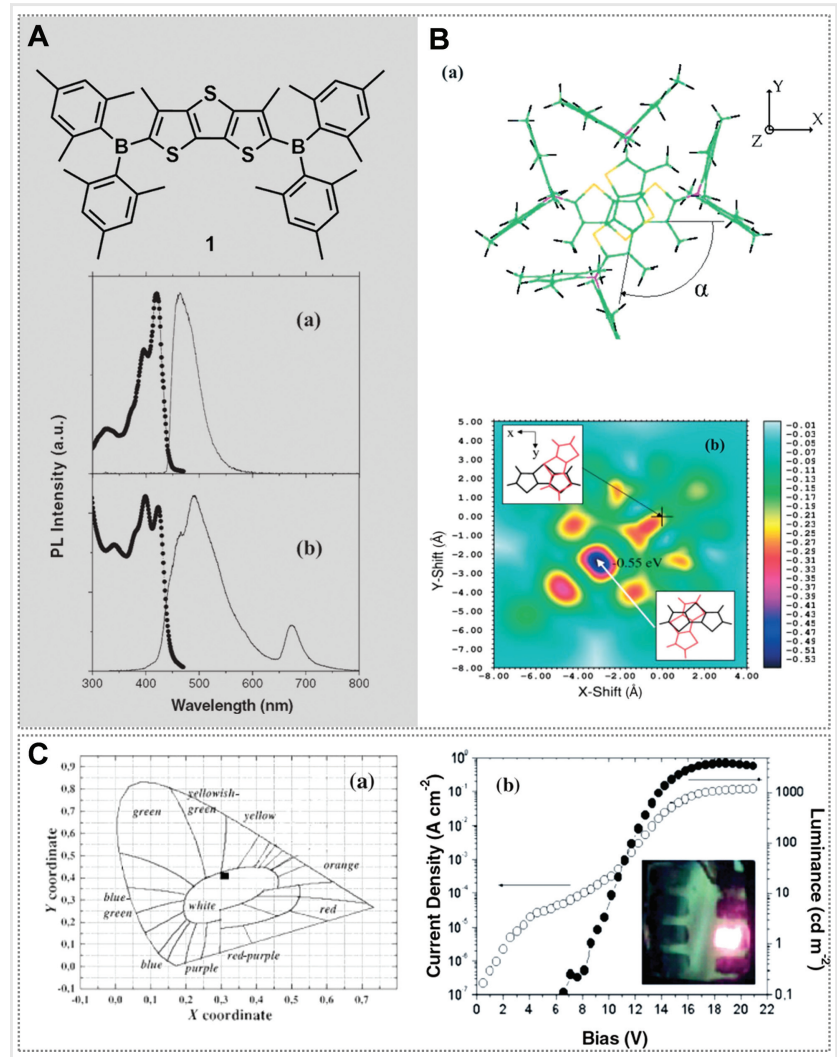

Figure 2 A) Molecular structure and UV-vis and PL of 3,5-dimethyl-2,6bis(dimesitylboryl)-dithieno[3,2-b:2', $\left.3^{\prime}-d\right]$ thiophene $\mathbf{1}$ in solution (a) and in the solid state (b). B) (a) Molecular structure of two interacting molecules forming a cross-like dimer. (b) Intermediated neglect of differential overlap/single configuration interaction (INDO/SCI) excitation energy shifts due to intermolecular interactions. The scale on the right corresponds to calculated excitation energy shifts. C) (a) CIE coordinates of EL emission. (b) Luminance and current density versus the applied voltage of the ITO/PEDOT:PSS/1/LiF/Al device. Inset: photo of a working OLED. Adapted with permission from Ref. 13. Copyright 2005 Wiley-VCH.
The borylated derivative was prepared by adding a solution of dimesitylboron fluoride in THF to a solution of 3,5-dimethyl-dithieno[3,2-b:2',3'-d]thiophene/BuLi in the same solvent. ${ }^{13}$ It is processable in common organic solvents and emits blue light in solution and white light in the solid state. By the aid of optical measurements, theoretical calculations and the comparison with structurally similar systems, we demonstrated that the white emission in the solid state is the result of the addition of the intrinsic blue-green emission of the isolated molecule with the red emission of cross-like dimers formed by selfassembly of $\mathbf{1}$ in the solid state, as shown in Figure 2B. The key element for the dimer formation is the boryl group, in which boron has a vacant p-orbital and then can act as an electron acceptor through $\mathrm{p}-\pi^{*}$ conjugation with adjacent electron-donor groups, particularly effective in the excited state.

There is extensive literature on the structural features and the peculiar electronic properties of boron derivatives. ${ }^{14}$ Using a spin-coated thin film of $\mathbf{1}$, a single-layer whiteemitting diode was fabricated. The electroluminescence (EL) spectrum showed similarity with the absorption spectrum in the solid state; however, the additional low-energy peak at $680 \mathrm{~nm}$ was much more intense, indicating a larger population in the aggregated state following electrical injection. The CIE (Commission Internationale de l'Éclairage) coordinates $(0.33,0.42)$ indicated a clear white light emission of the lightemitting diode (LED), characterized by high stability to the applied voltage (Figure 2C). Note that this is the first example of a white-emitting LED obtained from a single material spontaneously self-assembling in the solid state. Despite the great development of white LEDs having as active materials mixtures of compounds emitting different colors (blue/ yellow and blue/green/red emitters) under various conditions, there are still very few organic molecules capable of producing white EL under electroexcitation in organic LEDs. ${ }^{15}$ An intriguing example - very recently reported ${ }^{15 a}$ - concerns quinazoline-based white light emitters. The molecular structure of compound 2PQ - PTZ, having donor- $\pi$-acceptor configuration with quinazoline (PQ) as acceptor and phenothiazine (PTZ) as donor separated by a phenyl spacer, is shown in Scheme 4 together with the corresponding synthetic procedure.

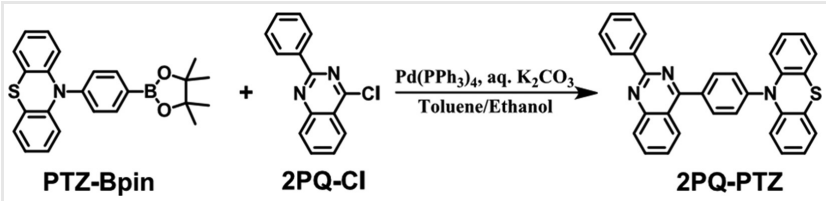

Scheme 4 Synthesis of quinazoline-based compound 2PQ-PTZ. Adapted with permission from Ref. 15a. Copyright 2020 American Chemical Society. 


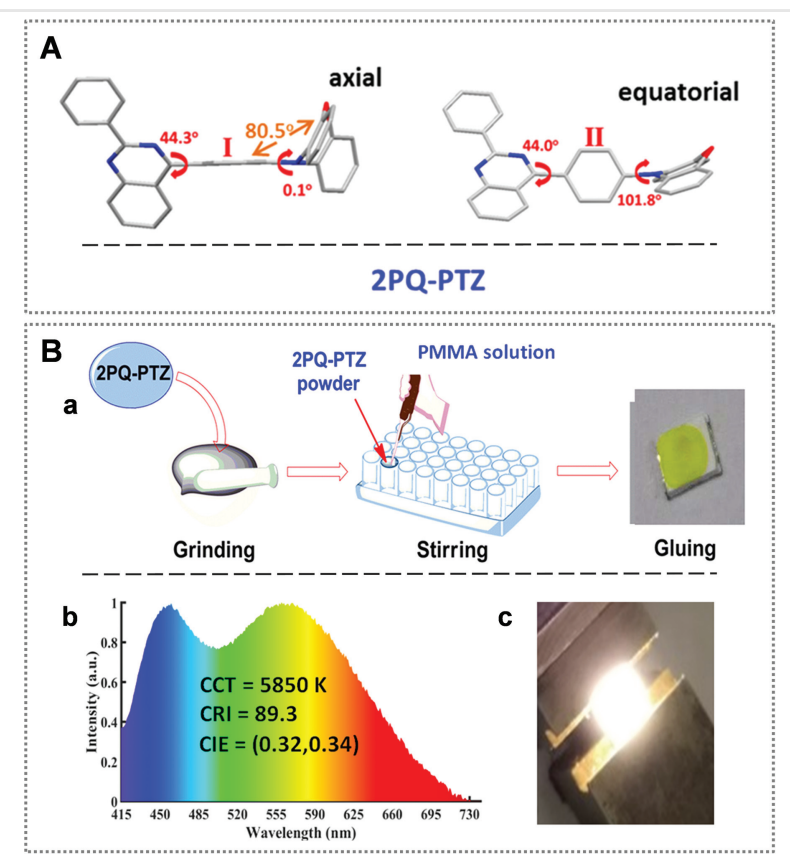

Figure 3 A) 'Axial' and 'equatorial' conformational isomers present in single crystals of 2PQ-PTZ. B) Characterization and photographs of white LED. (a) LED fabrication process. (b) Spectrum of LED of $2 \mathrm{PQ}$ - PTZ/PMMA onto the surface of LED chip. (c) Photographs of the white luminescence when LED is turned on. Adapted with permission from Ref. 15a. Copyright 2020 American Chemical Society.

In the single crystal of $2 \mathrm{PQ}-\mathrm{PTZ}$, two different conformational isomers, named by the authors 'axial' and 'equatorial', were found to coexist in a 1:1 proportion. The isomers are depicted in Figure 3A. According to the authors, the single crystal of $2 \mathrm{PQ}-\mathrm{PTZ}$ is the first experimental example of the coexisting (not interconverting) 'ax' and 'eq' conformers in rigid organic conjugated molecules. A detailed photophysical study of the two conformational isomers is described, supported by theoretical calculations. In thin films, using the appropriate matrix, 'ax' and 'eq' conformers emitted blue and orange light, respectively, providing complementary colors for white light emission. A white light-emitting LEDs based on 2PQ - PTZ and displaying 0.32, 0.34 CIE coordinates could be fabricated (panel B) with external quantum efficiency amounting to $10.1 \%$, which was the highest performance reported until then. ${ }^{15 a}$

\subsection{Light-Emitting Transistor and Bicolor Light- Emitting Diode from a Single Compound}

The molecular structure and the synthesis of the material in question, namely 2,6-bis-( $5^{\prime}$-hexyl-[2,2']bithiophen-5-yl)-3,5-dimethyl-dithieno[3,2-b;2',3'-d]thio-thiophene, DTT7Me, ${ }^{16}$ are reported in Scheme 5.

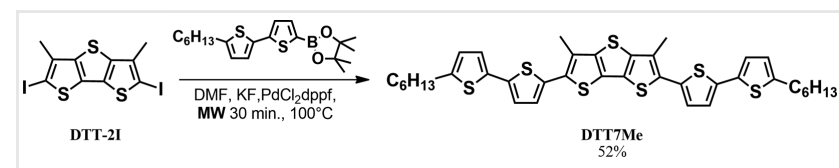

Scheme 5 Synthesis of 2,6-bis-(5'-hexyl [2,2']bithiophen-5-yl)-3,5dimethyl-dithieno[3,2-b;2',3'-d]thiophene, DTT7Me. Adapted with permission from Ref. 16. Copyright 2006 Wiley-VCH.

Based on our previous experience on thiophene oligomers, DTT7Me was designed in order to be soluble in common organic solvents, to possess great conformational flexibility and display good charge transport (because of the rigid dithienothiophene inner core) and good photoluminescence (PL; thanks to the presence of the methyl groups keeping the molecules sufficiently apart from each other). Exploiting the conformational flexibility and aggregation capabilities of this compound under different experimental conditions, a light-emitting transistor (LET) and a bicolor bipixelated light emitting diode could be fabricated.

\subsubsection{Light Emitting Transistor from DTT7Me}

A LET is a multifunctional device combining good charge transport with good EL. ${ }^{17}$ These conditions are not often simultaneously fulfilled since materials having high charge mobility generally also display efficient $\pi-\pi$ stacking, which in turn causes PL quenching because of nonradiative decay processes due to strong intermolecular interactions. A single-layer LET was prepared using both a vacuumevaporated and a drop-cast film of DTT7Me from toluene, both films displaying a high degree of order. Figure 4A $(a-c)$ shows the AFM images of vacuum-evaporated films of DTT7Me of different thicknesses on $\mathrm{SiO}_{2}$, while Figure 4A (d, e) shows laser scanning confocal microscopy (LSCM) fluorescence images of a 20 -nm-thick vacuum-evaporated film (d) and of a drop-cast film on $\mathrm{SiO}_{2}$ (e).

Both AFM and LSCM images show that the films are characterized by a high degree of surface coverage. The thickest vacuum-evaporated film is formed by large grains covering the entire surface of the substrate (c) and displays a spatially continuous red luminescent signal (d). Figure $4 \mathrm{~B}$ shows the X-ray diffraction profile of the crystalline powder of DTT7Me (c) together with the profile of the vacuumevaporated film of $20 \mathrm{~nm}$ thickness (a) and of the drop-cast film (b). The (c) profile indicates a high crystalline degree and a highly ordered structure of DTT7Me powders. Some of the peaks present in the powder profile are also found in the X-ray pattern of the cast film (b). On the contrary, none of the two peaks present in the profile of the vacuum-evaporated film is present in DTT7Me powder, indicating that the cast film (b) and the vacuum-evaporated one (c) belong to two different polymorphs, probably generated by different inter-ring angles of the terminal bithiophenes. The inset in panel $B$ 
shows the proposed model for the supramolecular organization of the vacuum-evaporated film. LETs were fabricated using vacuum-evaporated films, as well as drop-cast films were grown on gold source-drain electrodes. The output optoelectronic characteristics are shown in Figure 5A and B, respectively, where the left $y$-axis reports the drain-source current $I_{\mathrm{ds}}$ and the right axis the EL.

A

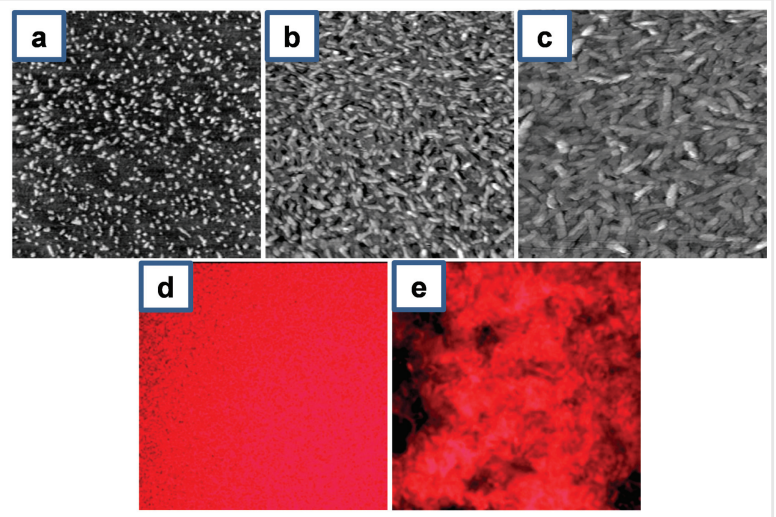

B

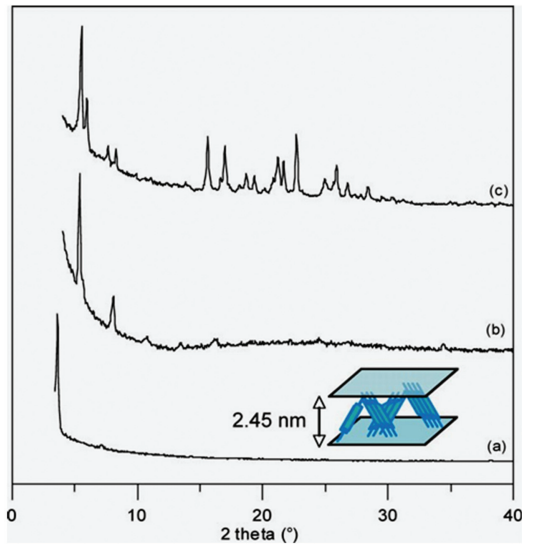

Figure 4 A) AFM micrographs $(4.5 \mathrm{~lm} \times 4.5 \mathrm{~lm})$ of DTT7Me films grown on $\mathrm{SiO}_{2}$ at a nominal deposition flux of $0.4 \AA \mathrm{s}^{-1}$ having nominal thicknesses of a) 3, b) 6 , and c) $20 \mathrm{~nm}$. The root-mean-square roughnesses of the films are $1.4,1.9$, and $2.5 \mathrm{~nm}$, respectively. Laser scanning confocal microscopy (LSCM) fluorescence images $(100 \mathrm{~lm} \times 100 \mathrm{~lm})$ of d) a 20-nm-thick vacuum-sublimed film and e) a drop cast film, on $\mathrm{SiO}_{2}$. B) XRD pattern of DTT7Me: a) a 20-nm-thick vacuum-sublimed film ( $F=0.4 \AA \mathrm{s}^{-1}$ ) on $\mathrm{SiO}_{2}$; b) a cast film on $\mathrm{SiO}_{2}$, and c) the precursor powder. Adapted with permission from Ref. 16. Copyright 2006 Wiley$\mathrm{VCH}$.

When the holes injected from the source electrode and moving along the channel meet the electrons near the drain electrode, they recombine radiatively in both cases and both intensity of emitted light and photocurrent increase with the increasing source-drain and gate voltages. Thus, both vacuum-sublimed and cast films generate EL under an appropriate applied voltage with very similar optical output characteristics. It is to be noted that this is the first example of an organic LET obtained by employing a non-polymeric material and the first having the active film obtained by drop casting.

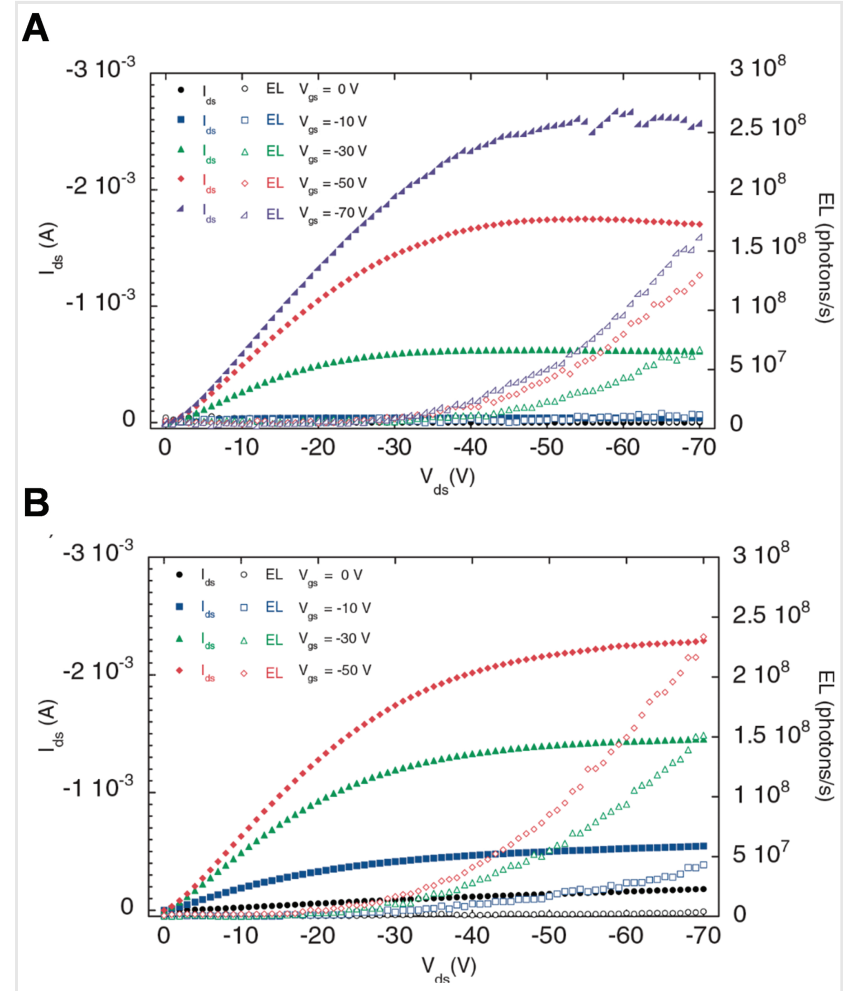

Figure 5 Optoelectronic output characteristics of DTT7Me-based OLETs. Drain-source current $\left(I_{\mathrm{ds}}\right)$ on the left $y$-axis, and EL on the right $y$-axis, vs. drain-source voltage $\left(V_{\mathrm{ds}}\right)$, recorded at various gate-source voltages $\left(V_{\mathrm{gs}}\right)$. A) Vacuum-sublimed film (thickness $20 \mathrm{~nm}, F=0.4 \AA \mathrm{s}^{-1}$ ), W/ $L=42,000 / 10(\mathrm{~lm} / \mathrm{lm})$. B) Drop-cast film, $W / L=42,000 / 6(\mathrm{~lm} / \mathrm{lm})$; $W=$ channel width, $L=$ channel length. Adapted with permission from Ref. 16. Copyright 2006 Wiley-VCH.

\subsubsection{Bicolor Organic Light-Emitting Diode from DTT7Me}

Using the same multifunctional compound, DTT7Me, presented in the previous paragraph and by means of an approach based on the surface-tension-driven (STD) lithography, ${ }^{18}$ a bicolor bipixelated LED was fabricated. ${ }^{19}$ Thanks to the STD technique, which allows the control of the molecular behavior of solutions at the solid/liquid interface, it was possible to simultaneously manipulate the conformation and the aggregation pattern of DTT7Me. By means of a single-step bottom-up procedure, it was possible to control both the conformation and self-assembly modalities of DTT7Me, hence the electro-optical properties of the compound in different positions of the substrate. Figure 6 illustrates step by step the procedure employed.

A drop of DTT7Me dissolved in toluene was deposited on a micrometer-sized grid, placed on a glass/ITO substrate, to induce geometrical confinement. During the evaporation of 
the solvent, the DTT7Me molecules move under and inside the meshes of the grid. The energy conditions at the different interfaces control molecular conformation and self-assembly, leading to two alternating emitting regions, orange and red, on which the bicolour pixelated organic LED was fabricated. ${ }^{19 \mathrm{~b}}$ Through AFM, confocal microscopy, X-ray diffraction of the patterned films and theoretical calculations, we were able to demonstrate that the red regions correspond to a crystalline film and a fully planar conformation of DTT7Me, while the green regions correspond to an amorphous film where DTT7Me is present as a series of distorted conformations of the terminal bithiophenes. ${ }^{19 a}$

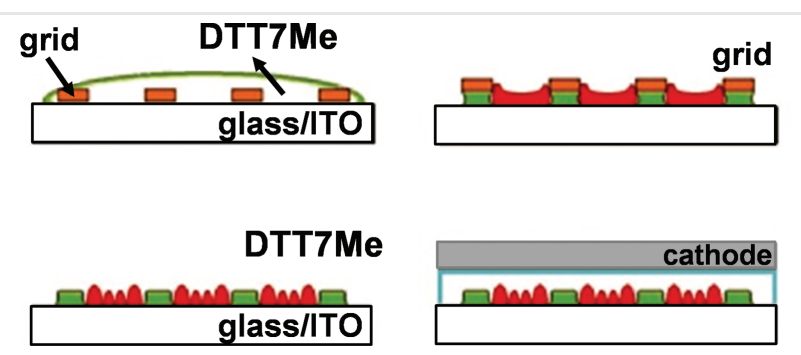

Figure 6 Sketch of the STD deposition technique used for the realization of the DTT7Me bicolor pixelated OLED. A drop of dilute solutions of DTT7Me is deposited on a template mesh, set on a glass/ITO substrate; during the solvent evaporation, the DTT7Me molecules move both under and inside the mesh; the interplay of the energetic conditions at the different interfaces drives the molecular self-assembly and realizes a bicoloured pixelated structure; the OLED is realized on the bicolor pattern. Adapted with permission from Ref. 19b. Copyright 2010 American Chemical Society.

Figure 7 (a, b) displays the structure of the fabricated bipixelated LED, i.e., the different layers composing the device, including the DTT7Me active layer, together with the corresponding energy level diagram. Figure 7c shows the scanning confocal microscopy image of the green and red patterns of the DTT7Me layer, while Figure 7d shows the threedimensional reconstruction of the spatially resolved PL emission. Finally, Figure 7e shows the PL spectra collected by confocal microscopy inside and under the grid mesh. The bicolor micro-pixelated LED of Figure 7 is the first example of a bicolor device obtained from a single molecular material employing a single-step procedure. Thanks to the conformational flexibility and spontaneous self-assembly properties of the thiophene oligomer DTT7Me, the formation of two regularly alternating regions on a micrometer scale emitting light of two different colors from a single material could be achieved.
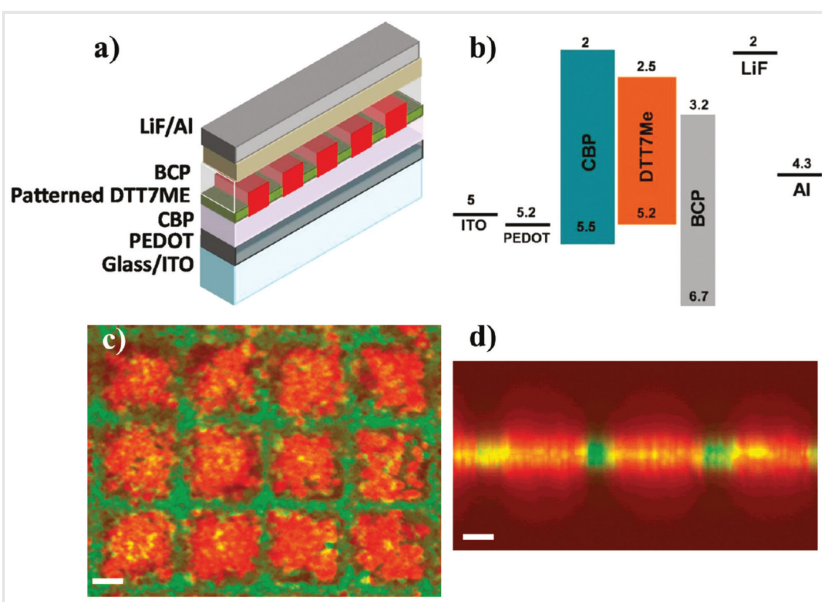

d)
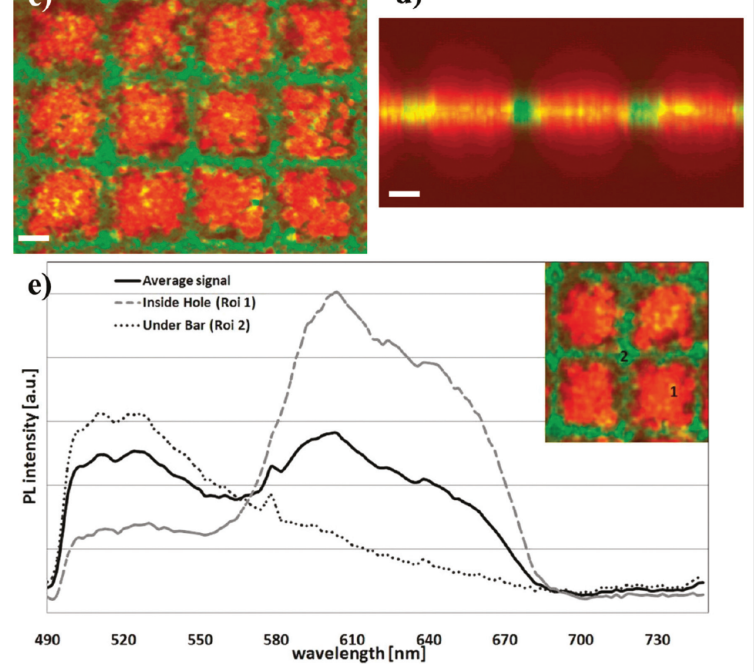

Figure 7 (a) Structure of the device ITO//PEDOT:PSS//CBP//DTT7ME// $\mathrm{BCP} / / \mathrm{LiF} / \mathrm{Al}$ and (b) the simplified energy level diagram. (c) Multichannel laser scanning confocal microscopy image of the DTT7Me layer of OLED patterned by STD technique. (d) 3D reconstruction of the spatially resolved PL emission, obtained by $x-z$ optical sections of confocal scans. (e) PL spectra collected by confocal microscopy in zones inside (dashed line) and under (dotted line) the grid mesh. The scale bars in (c) and (d) correspond to $10 \mu \mathrm{m}$. Reprinted with permission from Ref. 19b. Copyright 2004 American Chemical Society.

\subsection{Random Laser from T5COx}

Thanks to their relevant PL properties, thiophene-based materials have been thoroughly investigated as active materials in lasing applications. ${ }^{20}$ Ghofraniha et al. demonstrated that a functionalized quinquethiophene, namely $3,3^{\prime}, 4^{\prime}$ ",3"'"-tetra-cyclohexyl-3",4"-di(n-hexyl)-2,2':5',2":5",2"':5"' ',2"''-quinquethiophene-1", 1 "-dioxide (T5OCX) (Figure 8a), when confined in patterns with different shapes is capable of producing a tunable random laser emission. ${ }^{21}$ A random laser is an optical device based on an active molecular layer in which defects, aggregates or external beads act as scattering centers inducing light amplification. ${ }^{22}$ As depicted in Figure 8 , the patterning of the light emitter $\mathrm{T} 50 \mathrm{Cx}$, achieved by spontaneous molecular self-assembly driven by STD lithography, induces the formation of well-defined nanoaggregates in spatially restricted environments. Ring, stripe and pixel 
microarray structures were realized by depositing few microliters of a solution of $\mathrm{T} 5 \mathrm{OCX}$ in dichloromethane on different stamps used as geometrical constraints (Figure $8 \mathrm{~b}-\mathrm{j}$ ). This approach allowed tuning dimensions and distribution density of the nanoaggregates while permitting to finely control some of the physical parameters in a confined system. Additionally, changes in the temperature during the solvent evaporation process led to a further tuning of the size and spatial distribution of the aggregates. Indeed, faster (slower) evaporation rates result in smaller (larger) aggregates. The authors demonstrated that self-assembled T50CX nanoaggregates behave as scattering centers leading to the fabrication of one-component organic lasers without employing an external resonator and with the desired shape by using soft lithographic techniques (Figure 81-n). Particularly, improved lasing properties were achieved in devices in which more packed and larger supramolecular nanostructures were present.

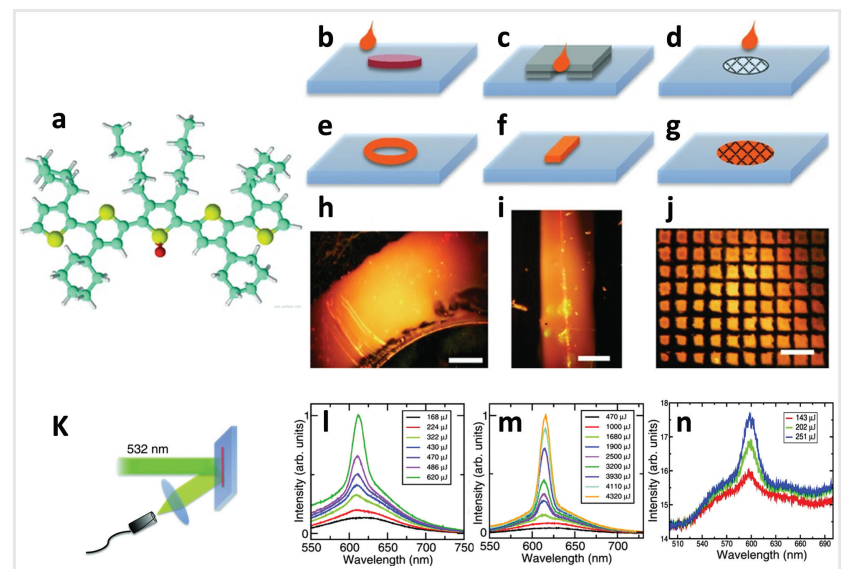

Figure 8 a) Schematic illustration of T5OCx. b-d) Sketches of the different bottom-up lithographic techniques employed to pattern the oligomer: ring (b, e), stripe (c, d) and squared pixels (d, g). h-j) optical images of the fabricated samples. A drop of $T 5 \mathrm{OC} x$ solution in $\mathrm{CH}_{2} \mathrm{Cl}_{2}$ is poured at the centre of the related template placed on a glass substrate. The average thickness of all samples is about $1 \mu \mathrm{m}$. Scale bars: $100 \mu \mathrm{m}(\mathrm{h}, \mathrm{j})$ and $50 \mu \mathrm{m}$ (i). K) A sketch of the random laser set-up. I-n) Emission spectra of a portion of the ring-shaped (I), stripe-shaped ( $m$ ), and single pixel (n). Adapted with permission from Ref. 21. Copyright 2013 Wiley-VCH.

\section{Supramolecular and Optoelectronic Properties of Oligothiophene-S,S-dioxides}

Oligothiophene-S,S-dioxides (OTOs) are OTs where one or more of the thiophene rings bear two oxygen atoms at the ipso-position, i.e., at the sulfur atom (see Scheme 1 ). ${ }^{23}$ The oxidation of sulfur in a substituted thiophene ring was first obtained by using the complex HOF. $\mathrm{CH}_{3} \mathrm{CN}$ (Rozen's reagent), prepared by passing $\mathrm{F}_{2}$ through a mixture of $\mathrm{CH}_{3} \mathrm{CN}$ and $\mathrm{H}_{2} \mathrm{O} .{ }^{24}$ Subsequently the oxidation was performed by using $m$-chloroperoxybenzoic acid as the oxidant and the preparation was extended to various OTOs (see, for example, Schemes 6 and 7). ${ }^{23 a}$

More recently, S-oxides (where sulfur bears only one oxygen) and mixed S,S-dioxides/S-oxides have been synthesized employing a novel methodology for the facile synthesis of oligo- and polythiophene-S-oxides and-S,S-dioxides under mild conditions by use of enabling technologies such as ultrasound and microwave assistance (Scheme 8). ${ }^{23 \mathrm{~b}}$

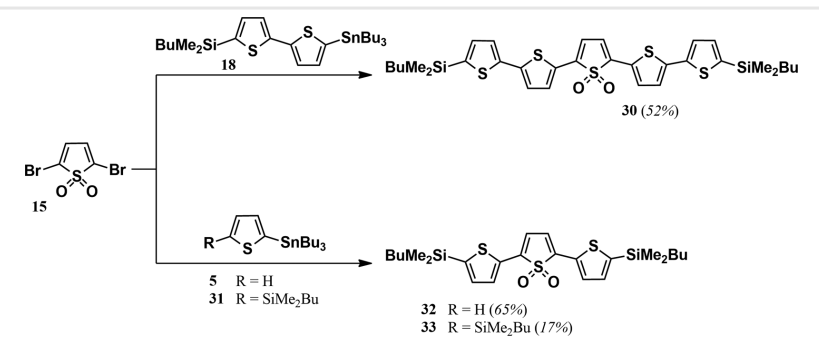

Scheme 6 Synthesis of oligothiophene-S,S-dioxide units containing one single inner thiophene-S,S-dioxide unit. Adapted with permission from Ref. 23a. Copyright 2004 American Chemical Society.

OTOs display smaller energy gaps, higher electron affinities and ionization energies than the corresponding OTs. ${ }^{25}$ Most OTOs are characterized by bright fluorescence in the solid state with high quantum yields (QYs), as shown in Figure 9 displaying the emission of cast films of some OTOs containing a single central thiophene-S,S-dioxide (TDO). ${ }^{26}$

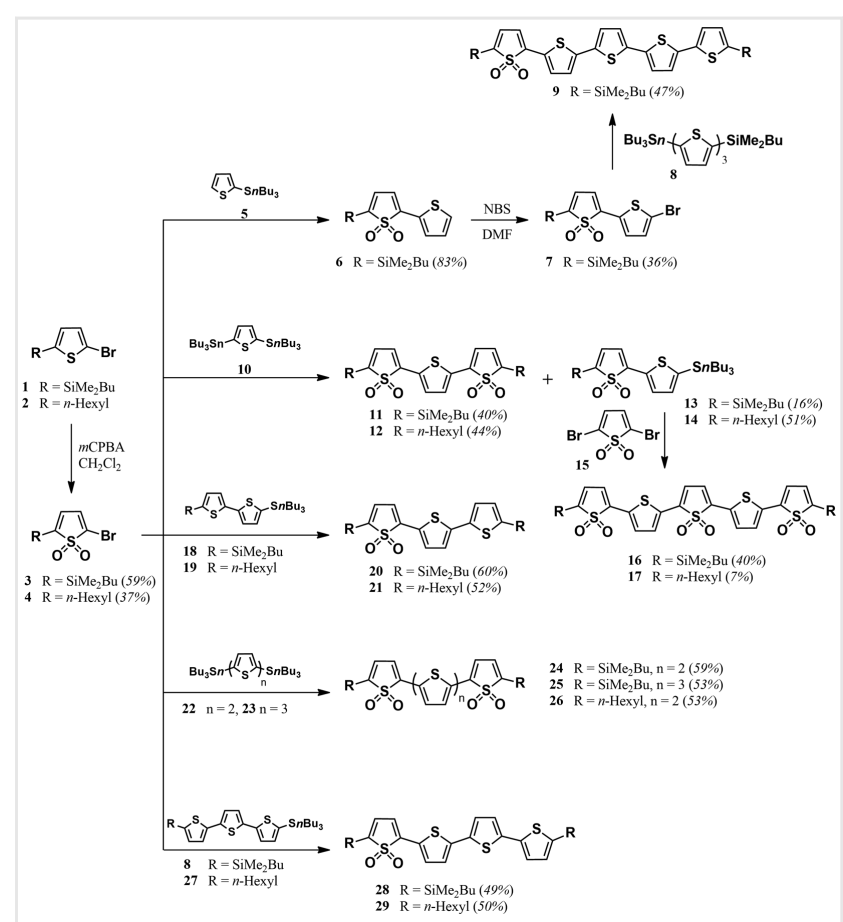

Scheme 7 Synthesis of dimers, trimers, tetramers, and pentamers containing from one to three thiophene-S,S-dioxide units. Adapted with permission from Ref. 23a. Copyright 1998 American Chemical Society. 
However, these compounds display a very low emission QY in solution and high emission QY in the solid state; hence, they are a special class of aggregation-induced emission materials that are nonemissive in solution but intensively emissive upon aggregation in the solid state. ${ }^{26}$ Concerning the relationship between supramolecular and optoelectronic properties, the most investigated so far are OTOs with one single central thiophene sulfonyl group. Structural modifications strongly impact on aggregation capabilities, which depend on the interplay of several intra- and intermolecular interactions. As a consequence, crucial features such as absorption and emission, redox properties, frontier energy levels and charge carrier mobility are modified with respect to the starting systems. While conventional OTs tend to aggregate mainly on the basis of $\pi-\pi$ stacking and van der Waals interactions, in TDOs, OTOs, the presence of the oxygen atoms generates a large number of intra- and intermolecular hydrogen bonds contributing to the packing forces and allows the molecules to stay away from each other. In consequence, most OTs show very low PL QYs in the solid state because of prevailing radiationless decay pathways caused by strong intermolecular interactions, while most OTOs display significant QYs in the solid state.

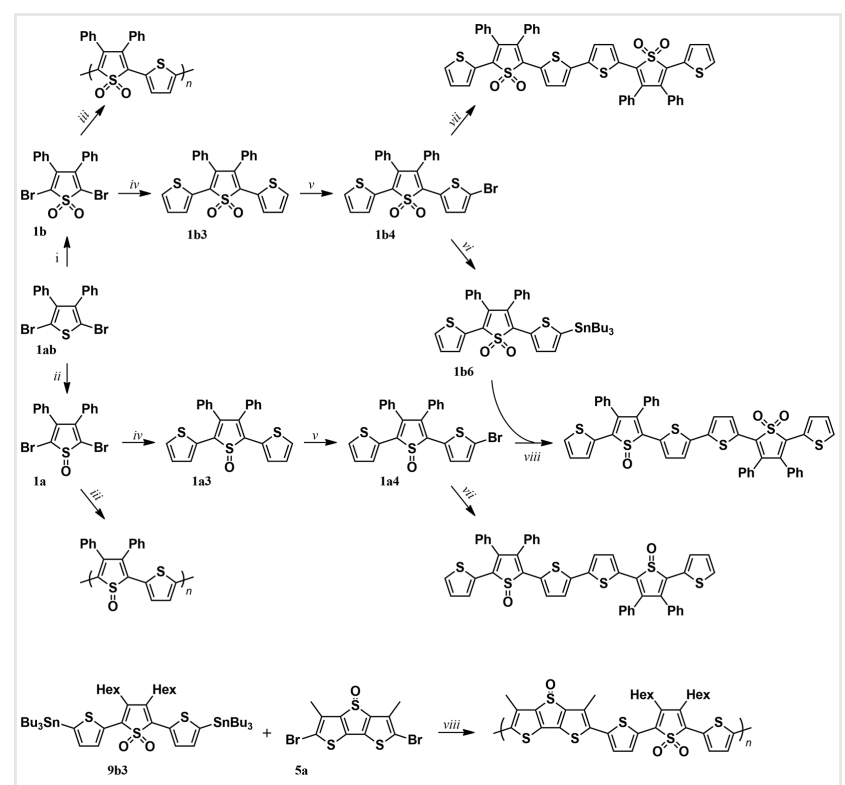

Scheme 8 Synthesis of oligo- and polythiophene-containing thiophene-S-oxide or thiophene-S,S-dioxide or mixed thiophene-S-oxide/S, S-dioxide units. Adapted with permission from Ref. 23b. Copyright 2016 Wiley-VCH. Reagent and conditions: i) 2 equiv $\mathrm{H}_{2} \mathrm{O}_{2} 30$ wt.\%, $\mathrm{CH}_{2} \mathrm{Cl}_{2}$ / $\mathrm{CF}_{3} \mathrm{COOH}(2: 1, \mathrm{v}: \mathrm{v})$; ii) 1 equiv $\mathrm{H}_{2} \mathrm{O}_{2} 30$ wt.\%, $\mathrm{CH}_{2} \mathrm{Cl}_{2} / \mathrm{CF}_{3} \mathrm{COOH}$ (2:1, v:v); iii) 2,5-bis(tributylstannyl)thiophene, $\mathrm{Pd}\left(\mathrm{PPh}_{3}\right)_{4}$, toluene, reflux overnight; iv) 2-(tributylstannyl)thiophene, $\mathrm{Pd}\left(\mathrm{PPh}_{3}\right)_{4}$, toluene, reflux overnight; $\left.v\right) \mathrm{N}$ bromosuccinimide (1 equiv), $\mathrm{CH}_{2} \mathrm{Cl}_{2} / \mathrm{CH}_{3} \mathrm{COOH}$ (1:1, v:v), US; vi) n-BuLi, $\mathrm{SnBu}_{3} \mathrm{Cl}, \mathrm{THF}_{(d r y)},-78^{\circ} \mathrm{C}$; vii) bis(pinacolato)diboron, $\mathrm{Pd}(\mathrm{dppf}) \mathrm{Cl}_{2}, \mathrm{NaHCO}_{3}$, $\mathrm{THF} / \mathrm{H}_{2} \mathrm{O}(2 / 1), \mathrm{MW}, 80^{\circ} \mathrm{C}$; viii) $\mathrm{Pd}\left(\mathrm{PPh}_{3}\right)_{4}$, toluene, reflux overnight.

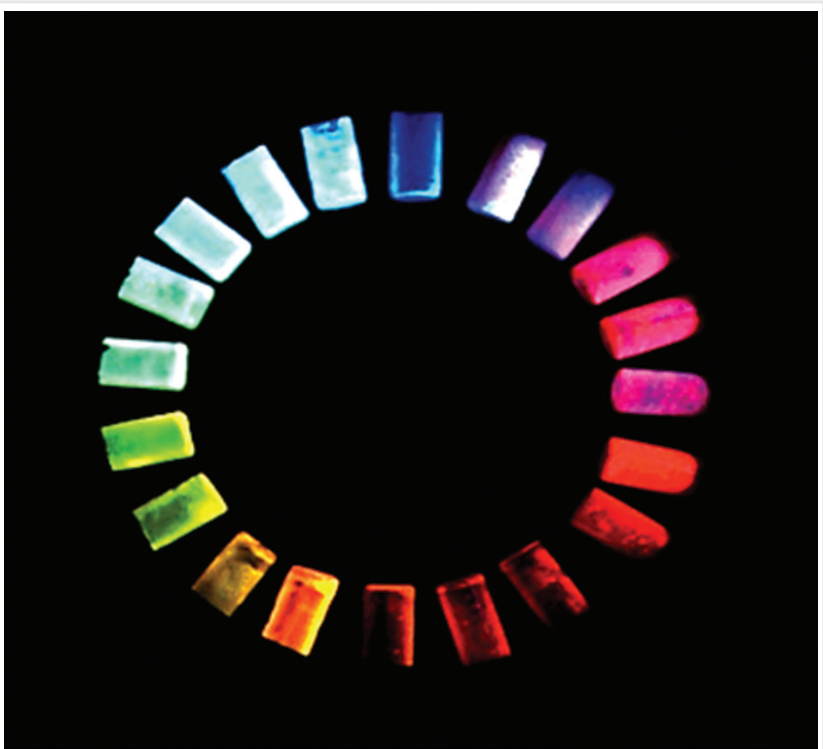

Figure 9 Cast films (from chloroform) of some oligothiophene-S,Sdioxides containing a single central thiophene-S,S-dioxide under UV excitation $\left(\lambda_{\text {exc }}=363 \mathrm{~nm}\right.$ ). Reprinted with permission from Ref. 26. Copyright 2000 American Chemical Society.

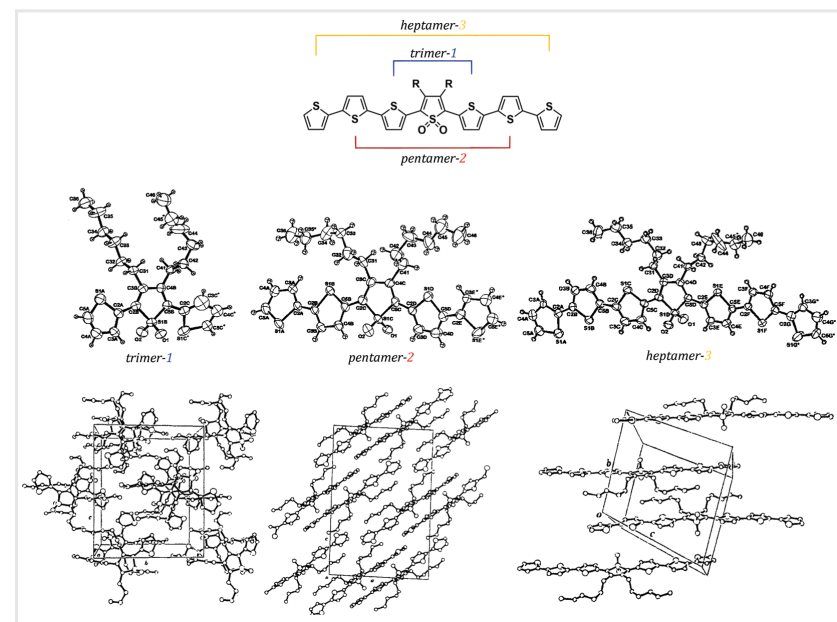

Figure 10 Molecular structure and perspective view of the crystal packing of trimer $\mathbf{1}$; molecular structure and projection of the crystal packing down the $b$-cell axis of pentamer 2; molecular structure and perspective view of the crystal packing of heptamer 3 . Adapted with permission from Ref. 28. Copyright 2000 American Chemical Society.

Figure 10 shows the single-crystal X-ray structures of trimer, pentamer, and heptamer having the same central alkylated TDO core but a different number of adjacent unsubstituted thienyl groups. Mainly due to the competing effects of dipolar $\mathrm{SO}_{2} \cdots \mathrm{SO}_{2}$ intermolecular interactions, intramolecular and intermolecular $\mathrm{C}-\mathrm{H}^{\cdots} \mathrm{O} \mathrm{O}$ hydrogen bonds and $S \cdots S$ interactions, compounds 1-3 display very different packing characteristics. In trimer $\mathbf{1}$ the molecules organize in a 
chiral form by spontaneous resolution and form a chiral single crystal. The conformation is very distorted, and the crystal packing shows a remarkable lack of short intermolecular contacts. Pentamer $\mathbf{2}$ displays a packing mode close to the 'herringbone' motif found in planar (or quasi-planar) conventional oligomers. In contrast to trimer $\mathbf{1}$, several very short van der Waals contacts $-C^{\cdots} \cdot \mathrm{C}, \mathrm{C} \cdots \mathrm{O}, \mathrm{S} \cdots \mathrm{S}$, rarely observed in OTs are present in it. The molecules of heptamer $\mathbf{3}$ are quasiplanar (except for the out-of-plane structure, due to the presence of the oxygen atoms of the sulfonyl group) and stack in parallel layers related by a crystallographic inversion center. There are many short $S \cdots O, S \cdots C, C \cdots O, C \cdots C$ and $S \cdots S$ intermolecular contacts, while layers of molecules interact via short $\mathrm{C}-\mathrm{H}^{\cdots} \mathrm{O}$ intermolecular contacts.

The absolute PL QYs of the crystalline powders of compounds 1, 2 and 3, (measured with an integrating sphere) are $0.45,0.12$ and 0.02 , respectively. The trend, which is different from that in solution, was accounted for by the supramolecular organization of the three compounds in single crystals, hence establishing a correlation between PL efficiencies and packing modalities. ${ }^{27}$ Contrary to the behavior in the solid state, in solution $\left(\mathrm{CH}_{2} \mathrm{Cl}_{2}\right)$ all three compounds display PL QYs amounting to less than $0.5,{ }^{28}$ as is the case of most OTOs with one single central TDO. The different PL properties of OTOs with respect to OTs are the result of the different molecular structures and supramolecular properties but also of the mechanisms for energy relaxation to the ground state. Photophysical and theoretical $^{29}$ studies show that sulfur dioxygenation causes a dramatic change in photophysics with respect to nonoxygenated thiophene oligomer. The relaxation dynamics to the ground state are related to conformational effects and solvent viscosity but not to solvent polarity despite the presence of the polar $\mathrm{SO}_{2}$ group. Moreover, of the two most important nonradiative decay mechanisms, internal conversion and intersystem crossing, only the former gives a significant contribution, contrary to what is observed in nonoxygenated oligomers. Strong intermolecular interactions in the solid state favor charge transport but are unfavorable to PL. An interesting compromise from the two opposing factors is found in the pentamer with one central dioxygenated unit and different alkyl substituents. The increase in electron affinity, induced by the dioxide functionalization in OTO, confers to charge carriers increased $n$-character thus allowing the fabrication of LEDs much more efficient than those obtained using conventional OTs. Multicolor LEDs with EL covering the entire visible range down to near-infrared were prepared using $\alpha$-quinquethiophene derivatives. ${ }^{30}$ Figure 11 shows the LED obtained using a thermally evaporated neat film of the $\alpha$-quinquethiophene-S,S-dioxide ${ }^{31}$ T5COX (see Section 2.3) using the $p-i-n$ technology, based on the deposition of highly conductive $p$ - and $n$-doped transport layers. $^{32}$ The doping of the $p$ and $n$ transport materials is realized by means of electron acceptor molecules, such as 2,3,5,6-tetrafluoro-7,7,8,8-tetracyanoquinodimethane
(F4TCNQ), and electron donor alkali metals, such as lithium or cesium (Figure 11A). As shown by the data reported in Figure 11 (B, C), luminances of $10,000 \mathrm{~cd} / \mathrm{m}^{2}$ were obtained with a voltage of $9 \mathrm{~V}$, about 30 times the best value reported for a bilayer configuration. Moreover, aging measurements showed device lifetimes of about 108 and 2200 hat 100 and $3200 \mathrm{~cd} / \mathrm{m}^{2}$ of starting luminances, respectively.

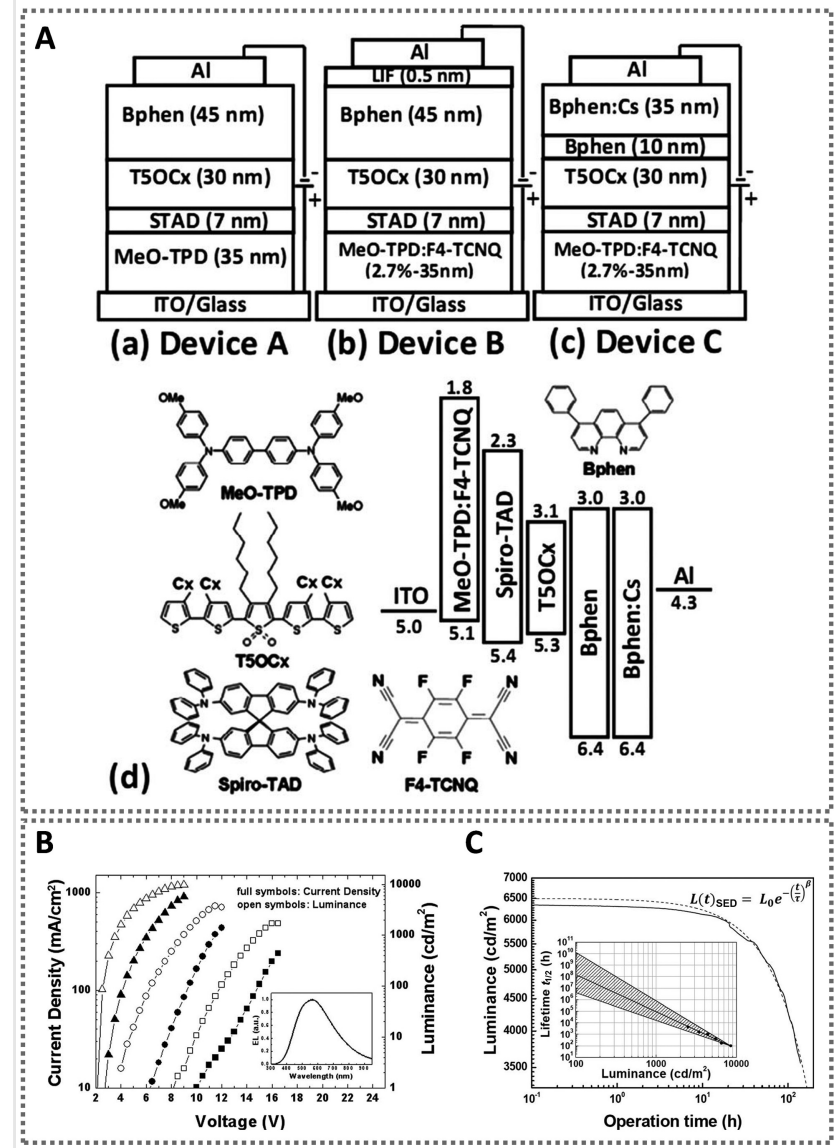

Figure 11 A) Structures of the tested OLEDs: (a) undoped device, (b) $p$-doped device, and (c) $p$-i-n device. (d) Energy levels of the $p$-i-n device and chemical structures of materials used. B) Current density (full symbols) and luminance (open symbols) vs. voltage for device $A$ (square), device $B$ (circle), and device C (triangle). Inset: EL spectrum of device C. C) Experimental lifetime curve starting from $6500 \mathrm{~cd} / \mathrm{m}^{2}$ (continuous curve), SED extrapolation (dashed curve); shown in the right corner is the SED equation. $L(t)$ is the luminance at time $t, L_{0}$ is the initial luminance, $t$ coincides with the decay time, and $\beta$ is called the dispersion factor, which is related to the shape of the curve; this last parameter does not vary for different current densities measured in the same structure. The halflifetime $t_{1 / 2}$ is related to $t$ by the equation: $t_{1 / 2}=(\tau / \beta) \ln 2$. Inset: lifetime vs. luminance; SED-extrapolated lifetime at different starting luminance (full symbols) and accelerated lifetime extrapolation (continuous curve) in the middle of the filled area. The filled area envelopes the incertitudes of the extrapolated data. Adapted with permission from Ref. 31a. Copyright 2009 American Institute of Physics.

Recently, a major contribution to the understanding of the optoelectronic and charge transport properties of 
dioxygenated OTs has come from Campos and coworkers. ${ }^{33}$ Choosing a model series of oligomers characterized by one to four TDO groups flanked on each side by a terminal thiophene, they were able to demonstrate that the prevailing charge carriers change from holes to electrons as the number of TDO units present in the backbone increases. $^{33 a}$ Along the series from the trimer to the octamer, the absorption maximum is progressively redshifted and the energy gap decreases from 2.1 to $1.4 \mathrm{eV}$. However, this large decrease is mainly due to the LUMO orbital, the HOMO remaining almost unchanged. In consequence, on passing from the trimer to the octamer, the nature of the prevailing charge carrier changes from $p$ - to $n$-, i.e., from holes to electrons as the number of TDO units present in the oligomer increases. It is to note that the control of charge carriers obtained on varying the length of the oligomer was unprecedented. The flexibility of the thiophene ring coupled to its ipso- di-oxidated and monooxidated counterparts is confirmed by our recent results ${ }^{23 b}$ by means of the fabrication of thin-film field-effect transistors demonstrating that while a conventional polythiophene has prevailing $p$-type charge transport characteristics, the corresponding polymer bearing alternating thiophene-S-oxide groups has ambipolar charge transport properties, whereas the corresponding polymer with TDO groups has prevalently $n$-type charge transport carriers.

\section{Colloidal Nanoparticles formed by Self-Assembly of Thiophene-Based Polymers}

In the field of chemical nanotechnology, an important role is played by nanoparticles (NPs) obtained by selfassembly of functional $\pi$-conjugated polymers for their biological applications - in particular as carriers for the delivery of therapeutic molecules - and for their use in organic electronics. ${ }^{34}$ Recently, NPs of poly(3-hexylthiophene), P3HT-NPs, have successfully been employed for in vitro and in vivo applications. ${ }^{35}$ The successful use of colloidal P3HT-NPs in field-effect transistors has also been reported. ${ }^{36}$ Below we describe two recent examples from our own work concerning the preparation and the optoelectronic characterization of novel thiophene-based oxygenated NPs (Section 4.1) and application of NPs of P3HT-NPs in flexible solid-state electrochromic devices (ECDs; Section 4.2).

\subsection{Spherical Nanoparticles of Poly(3-hexyl) thiophene Containing Thiophene-S,S-dioxide Units}

Di Maria et al. ${ }^{37}$ have described the preparation and optoelectronic characterization of novel NPs of P3HT containing variable amounts of TDO units. The NPs were prepared either by first synthesizing P3HT NPs and then oxygenating the NPs with Rozen's reagent ${ }^{24}$ or by first oxygenating P3HT with Rozen's reagent and then preparing the NPs, as illustrated in Scheme 9.

In both cases, the NPs were prepared by the nanoprecipitation method, i.e., by first dissolving the polymer in THF (in the absence of surfactants) and then adding this solution into distilled water under rapid stirring. ${ }^{38}$ The different preparation modalities afforded different NPs. Those obtained from oxygenation of preformed NPs of P3HT were core-shell NPs (P3HT@PTDO-NPs in Scheme 9) having the oxygenated polymer arranged in the external shell and the non-oxygenated polymer in the inner core. On the contrary, those obtained after oxygenation of P3HT (PTDONPs in Scheme 9) had the oxygen randomly distributed inside and outside the entire volume of the NPs. By employing in both cases variable amounts of the oxidant, a fine tuning of NP dimensions and optoelectronic properties was achieved.

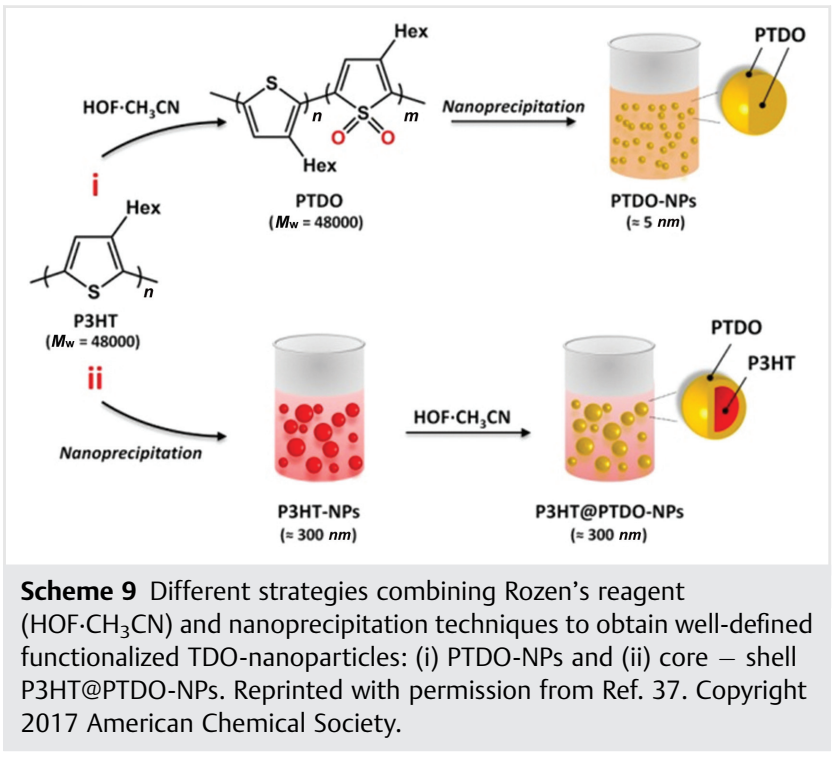

Scheme 9 shows that the dimensions of PTDO-NPs could be modulated in the range of $60-150 \mathrm{~nm}$, becoming progressively smaller as the oxygenation degree of the polymer increased, a result probably due to different interand intramolecular interactions promoted by oxygen during NP formation. By contrast, the dimensions of P3HT@PTDONPs, obtained by oxygenation of preformed P3HT-NPs, were always nearly $300 \mathrm{~nm}$. The $z$-potential values indicated that the stability of the colloidal solutions of NPs increased as the number of TDO groups present in the NPs increased, the solutions being stable for months without any sign of precipitation. Transmission electron microscopy and AFM images reported in Figure 12 (panels A $[a, b]$ and $B[a, b]$ ) show the spherical morphology of the NPs. 

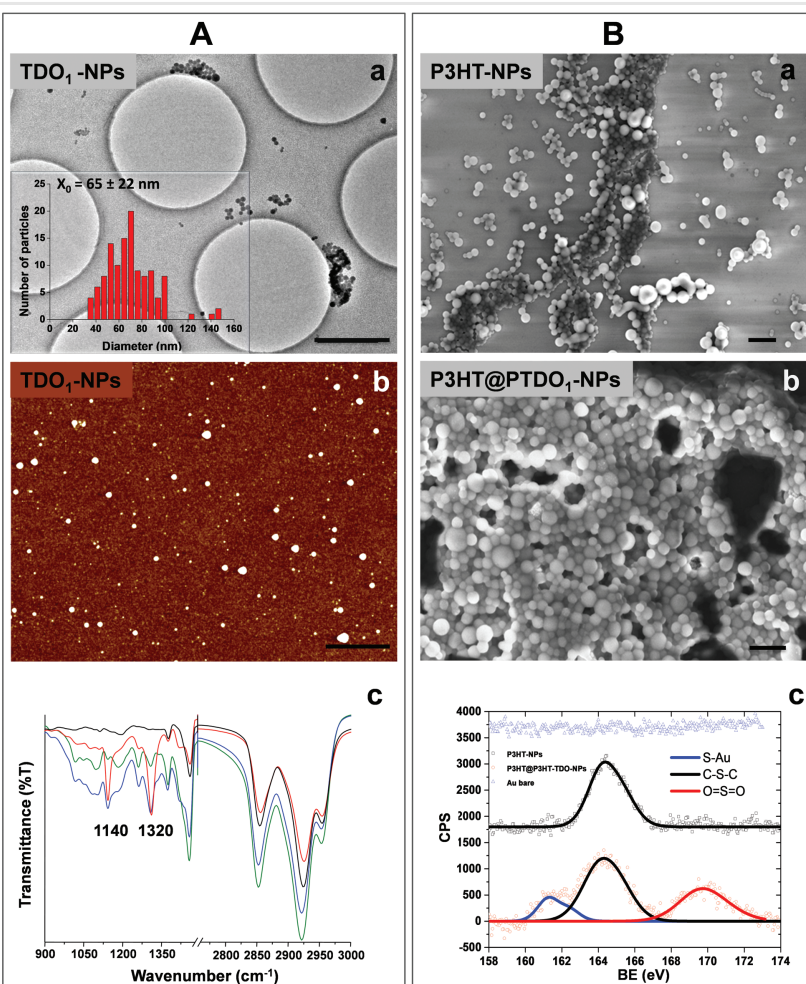

Figure 12 A) (a) Low-magnification TEM micrograph of a PTDO ${ }_{1}-N P$ sample with its particle size histogram in the inset. (b) AFM image of $\mathrm{PTDO}_{1}$-NPs. (c) Infrared spectra in the region between 900 and $1400 \mathrm{~cm}^{-1}$ and $2750-3000 \mathrm{~cm}^{-1}$ of PTDO-NPs $\left(\right.$ PTDO $_{0.25}$-NPs, green line; $\mathrm{PTDO}_{0.5}-\mathrm{NPs}$, blue line; $\mathrm{PTDO}_{1}$-NPs, red line) compared to P3HT-NPs (black line). B) SEM image of P3HT-NPs (a) and P3HT@PTDO 1 NPs (b) obtained after addition of 1 equiv of HOF. $\mathrm{CH}_{3} \mathrm{CN}$ to P3HT-NPs. (c) X-ray photoelectron spectra of P3HT-NPs ( $\square$ ) and P3HT@ PTDO $_{1}$-NPs (०). Scale bar, $1 \mu \mathrm{m}$. Adapted with permission from Ref. 37. Copyright 2017 American Chemical Society.

The figure also displays the IR spectra and X-ray photoelectron spectra of PTDO-NPs and P3HT@PTDO-NPs obtained with the maximum amount of oxidant employed. The IR spectra show the presence of the peaks typical of TDO units present in OTOs hence confirming that the oxygenation has occurred at the sulfur atom (panel A[c]). Also, X-ray photoelectron spectroscopy measurements are in agreement with the proposed structure for the different types of NPs (panel $\mathrm{B}[\mathrm{c}]$ ). Indeed, while the spectrum of pristine P3HT-NPs displays only one peak corresponding to the binding energy of the $\mathrm{S}-\mathrm{C}$ bond, the spectrum of core shell P3HT@PTDO-NPs displays three peaks centered at different binding energies and indicating the presence of three non-equivalent sulfur atoms, one due to metal-sulfur interactions and the other two corresponding to the oxidation of the thiophene sulfur atom. The control of the oxygenation degree also allows the fine modulation of the optical properties of the NPs. Passing from P3HT-NPs to PTDO-NPs, a blue-shift of the maximum wavelength absorption was observed - ascribed to the smaller size of the NPs causing a decrease in polymer conjugation length paralleled by a progressive decrease in the intensity of fluorescence emission as the amount of oxygen increased. On the contrary, going from P3HT-NPs to core-shell P3HT@PTDO-NPs, only small variations in the UV-vis and fluorescence spectra are observed, in agreement with the fact that oxygenation is limited to the outer shell of the NPs.

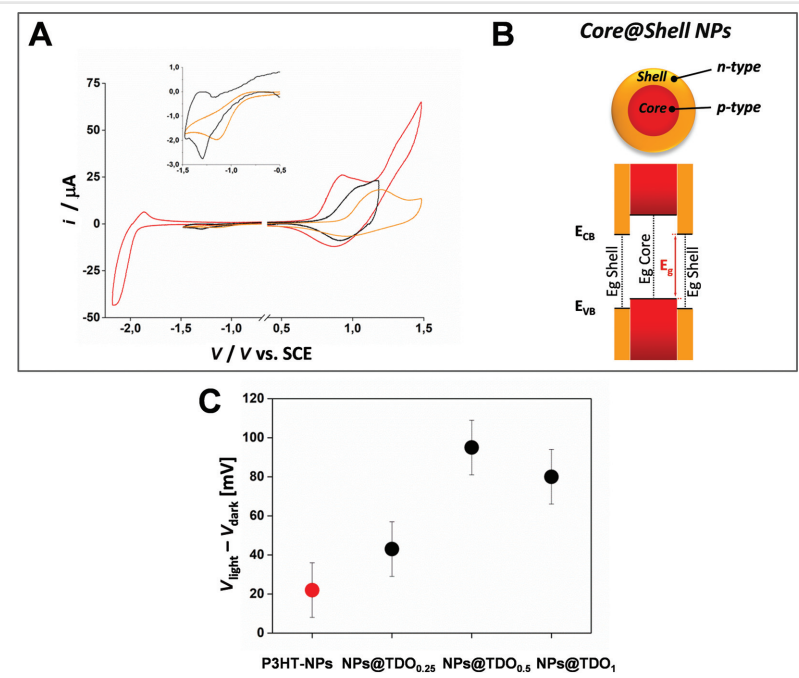

Figure 13 (A) Cyclic voltammetries of P3HT-NPs (red line), P3HT@PTDO-NPs (black line), and PTDO-NPs (orange line). (B) Schematic representation of the cross-section of core - shell P3HT@PTDO NPs made of PTDO in the shell and P3HT in the core and its corresponding energy levels. (C) Measured surface photovoltage values corresponding to different nanoparticles (red circle, P3HT-NPs; black circle, P3HT@PTDO-NPs). Adapted with permission from Ref. 37.

Copyright 2017 American Chemical Society.

Figure 13 compares the cyclovoltammetries of P3HT-NPs, PTDO-NPs and P3HT@PTDO-NPs. The figure shows that the different structures and morphologies of the NPs have a striking effect on their electrochemical properties (panel A). The data show that the structuration into NPs causes the decrease (on the order of hundreds of $\mathrm{mV}$ ) of the HOMOLUMO energy gap with respect to the starting polymer. More importantly, both the oxidation and reduction potentials are largely affected by the number of TDO units present in the NPs. In PTDO-NPs, as the number of TDO units increases, the oxidation potential increases while the reduction potential becomes less negative, as already observed in OTOs, ${ }^{25 a}$ leading to low band-gap NPs. By contrast, in core-shell P3HT@PTDONPs, the increasing oxygenation of the external shell causes only a negligible variation in the oxidation potential but a remarkable change in the reduction potential which becomes progressively less negative. This is in agreement with the fact that the inner core of the NPs is made of pure P3HT and only a few external layers are oxygenated. Consequently, the energy 
gap of core-shell P3HT@PTDO-NPs is lower than that of PTDONPs and P3HT-NPs (panel B). At the maximum oxygenation degree, P3HT@PTDO-NPs displayed an energy gap of $1.42 \mathrm{eV}$ while PTDO-NPs at maximum oxygenation displayed an energy gap of $2.67 \mathrm{eV}$, both to be compared to the value of pure-shell P3HT-NPs, $1.54 \mathrm{eV}$. The high electron affinity of P3HT@PTDO-NPs suggests that they may display ntype semiconductor behavior, in line with what has already been observed in polythiophene-S,S-dioxides. ${ }^{23 b, 33 a}$ Kelvin Probe measurements, also reported in Figure 13C, confirm this to be the case. Kelvin Probe measurements allow the simultaneous investigation of the structural and electronic properties of a surface with nano-scale resolution. ${ }^{39}$ The formation of charges is revealed by a shift of the surface photovoltage under illumination with respect to the dark. Charge carrier generation and separation under illumination, after dissociation of excitons, were measured for the three types of NPs. Figure 13C shows the data for P3HT@PTDO-NPs with different oxygenation degrees and, for comparison, also for non-oxygenated P3HT-NPs. It is seen that in core-shell P3HT@PTDO-NPs the parameter $V_{\text {light }}-V_{\text {dark }}$, i.e., approximately the charge density of the NPs, increases with the oxygenation degree up to a maximum of nearly $100 \mathrm{mV}$ (five times the value for P3HT-NPs), suggesting that beyond a given oxygenation degree in the outer shell no better charge separation may be achieved.

\subsection{Spherical Nanoparticles of Poly(3-hexylthiophene- 2,5-diyl) for Electrochromic Devices}

Electrochromism is a reversible change of the color of materials under an applied external voltage. ${ }^{40}$ Conjugated polymers are among the most important electrochromic materials for application in devices. In turn, polythiophenes are amongst the most studied electrochromic polymers, owing to their significant color contrast, considerable conductivity, chemical stability, and color tunability obtained by structural changes via organic synthesis. Several polythiophenes with tailored electrochromic properties have been reported giving good performance in devices, in particular poly(3,4-ethylenedioxythiophenes) and its derivatives. ${ }^{41}$ It is known that the morphology of the active film may strongly affect the performance of ECDs. In particular, improved results are obtained employing nanostructured thin films. ${ }^{42}$ We found that employing nanostructured films obtained by deposition of P3HT-NPs by spread-casting, it was possible to fabricate ECDs of P3HT displaying improved performance with respect to what was obtained with cast films from chloroform (Figure 14). ${ }^{43}$ The P3HT samples employed for the fabrication of the ECDs were prepared with the same methodology employed for the preparation of highly pure P3HT-NPs employed as fluorescent probes for cellular studies. ${ }^{44}$ Figure 14 shows the characterization of P3HT obtained by oxidative polymerization of 3-hexyl-thiophene together with a sketch illustrating the formation and the centrifugation of the NPs obtained by nanoprecipitation (panels $\mathrm{A}$ and $\mathrm{B}$ ). In the proton spectrum, the peaks pertaining to the four triads characteristic of $\mathrm{P} \mathrm{HT}^{44,45}$ are visible.

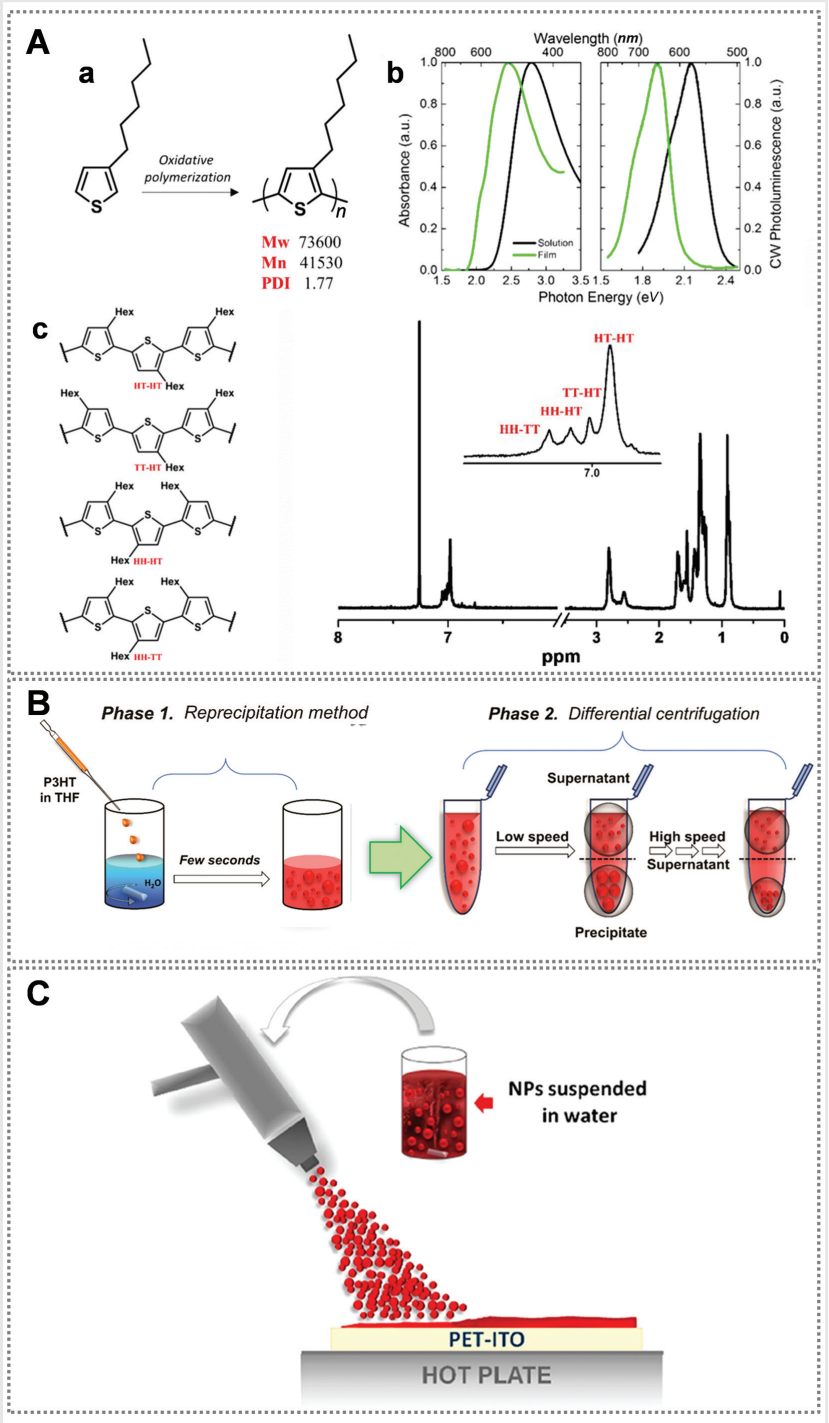

Figure 14 A) (a) Synthesis of P3HT by oxidative polymerization with $\mathrm{FeCl}_{3}$. (b) Normalized PL and UV-Vis spectra. (c) ${ }^{1} \mathrm{H}-\mathrm{NMR}$ spectra in $\mathrm{CHCl}_{3}$ showing the presence of the different configurational triads. Reprinted with permission from Ref. 44 (Figure S1). Copyright 2017 Royal Society of Chemistry. B) Preparation of P3HT-NPs by use of the reprecipitation method and differential centrifugation. Adapted with permission from Ref. 44. Copyright 2017 Royal Society of Chemistry. C) Spray-casting of the water dispersion of P3HT-NPs on a flexible PET-ITO substrate by means of an air-gun sprayer. Adapted with permission from Ref. 43. Copyright 2020 American Chemical Society.

Grazing-incidence wide-angle X-ray scattering measurements on P3HT-NPs of 100, 200, and $400 \mathrm{~nm}$ size spray coated on silicon support showed that large, randomly oriented, 
crystalline domains were present in all NPs, independent of their size. ECDs were fabricated employing spray-casted films from P3HT-NPs suspensions in water deposited on flexible PET-ITO substrates with the aid of an air-gun sprayer (see Figure 14C) and compared to analogous devices employing P3HT cast films from chloroform. All devices show reversible color transitions between red and light blue by applying a $-1.5 / 1.5 \mathrm{~V}$ voltage, as shown in Figure 15A.

A

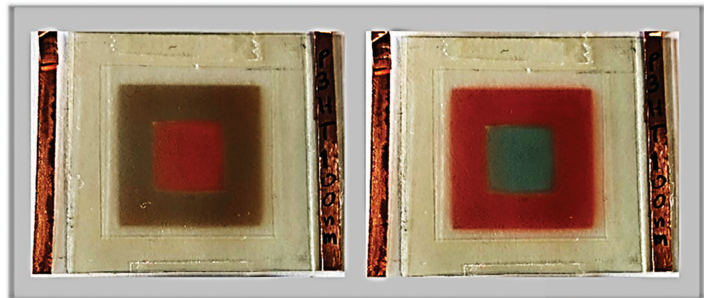

B
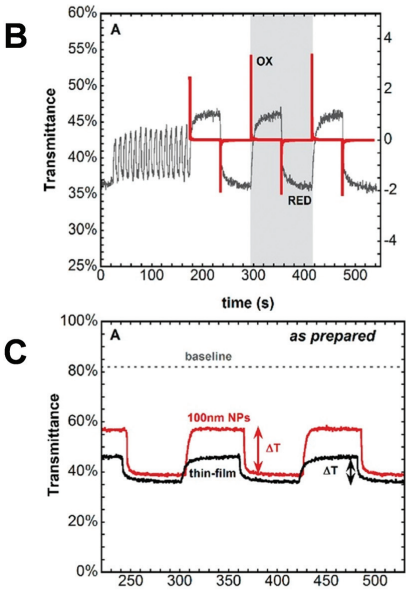

$t(s)$
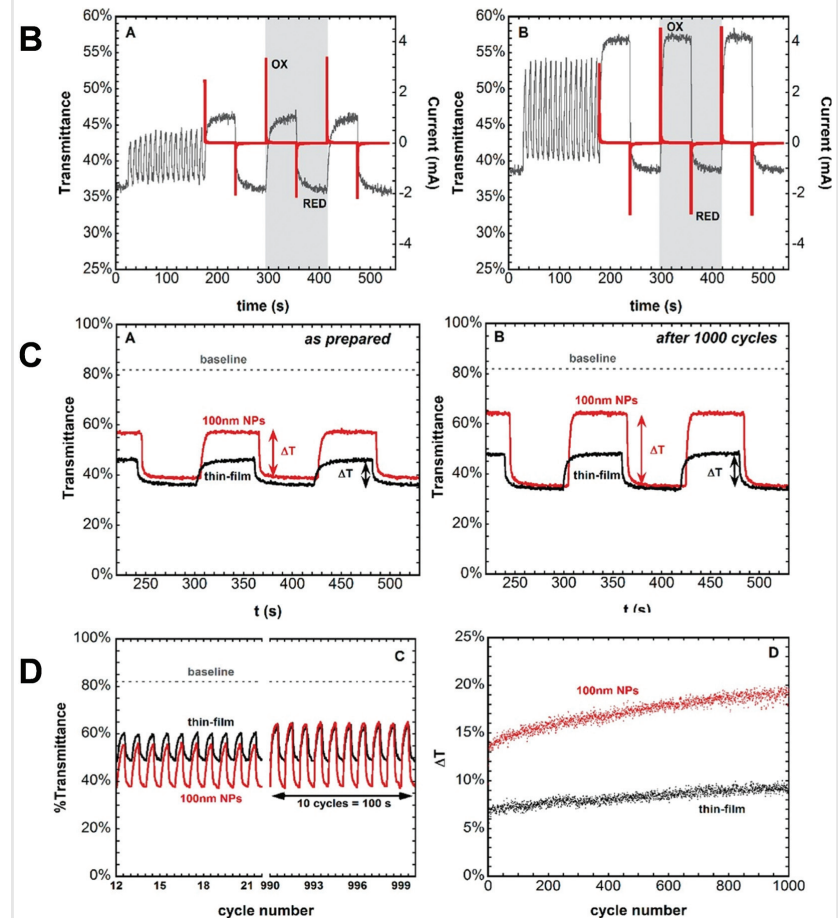

Figure 15 A) Electrochromic device with spray-coated $100 \mathrm{~nm}$ P3HTNPs at different redox states. Inner square reduced (left) and oxidized (right) by applying1.5/-1.5 V. B) (A, B) Transmittance (black) and current (red) data obtained from the characterization of the device using P3HT as cast film and P3HT-NPs of $100 \mathrm{~nm}$, respectively. C) (A, B) Comparison in transmittance of the devices using $\mathrm{P} 3 \mathrm{HT}$ thin film and P3HT-NPs (100 nm), as prepared and after 1000 cycles, respectively. D) (C) Changes in transmittance of P3HT thin film and P3HT $100 \mathrm{~nm}$ NP devices during the cycling measurement and (D) Optical contrast evolution (in $\Delta T$ ) of the P3HT thin film and P3HT $100 \mathrm{~nm}$ NP devices during the 1000 cycles performed. In all measurements, $1.5 \mathrm{~V}$ was used for oxidation and $-1.5 \mathrm{~V}$ was used for reduction $(1 \mathrm{cycle}=10 \mathrm{~s}$ ). Adapted with permission from Ref. 43. Copyright 2020 American Chemical Society.
Performance parameters were evaluated on the basis of switching time, charge consumption $(Q)$, optical contrast $(\Delta T)$ and improved durability (cycling). It was found that devices fabricated with P3HT-NPs with size of $100 \mathrm{~nm}$ displayed enhanced properties with respect to ECDs having P3HT-NPs of larger size as well as with respect to ECDs with thin films deposited from chloroform.

Figure 15B shows a plot of transmittance and current data from the device employing a thin film of P3HT and the device with a film of NPs of $100 \mathrm{~nm}$, the data being largely in favor of the latter. The same is true for the comparison in transmittance of the devices as prepared and after 1000 cycles (panel $\mathrm{C}$ ) and changes in transmittance during the cycling measurement or optical contrast evolution during the 1000 cycles performed (panel D). Finally, a significant result of this work is that employing films of NPs of P3HT dispersed in water is not only a way to obtain more performant ECDs but also an environmentally sustainable procedure, avoiding toxic organic solvent vapors for the users and with a much lower environmental impact.

\section{Conclusions and Outlook}

In this short review, we have reported a few examples taken from our own work aimed to show that thiophene derivatives are a rich source of molecular entities whose supramolecular aggregation allows the fabrication of a variety of highly performant functional optoelectronic devices. Thiophene derivatives can generate a multiplicity of supramolecular structures, hardly predictable on the mere structure of the molecular components but programmable through the use of molecules containing sufficient information in their covalent framework to promote their spontaneous supramolecular organization into functional superstructures via non-covalent interactions (noncovalent interactional algorithms according to J. M. Lehn's definition ${ }^{46}$ ), as in the case of fluorescent and conductive nanostructured microfibers. ${ }^{47}$ Thus, to face up to new opportunities in the field of supramolecular optoelectronic materials, there is an urgent need for innovative thiophene-based compounds capable to give rise to supramolecular architectures with programmed functions. In parallel, new synthetic methodologies should be developed through more ecofriendly and lowcost procedures such as, for example, use of aqueous solvents and catalysts without polluting metals. Finally, in the present context, it should be reminded that besides the works concerning thiophene derivatives - from ourselves and the many researchers working in the field - there is plenty of excellent examples of supramolecular structures and applications from other classes of conjugated oligomers and polymers. Nevertheless, the peculiarity of thiophene derivatives stems from their numerous applications in biological and medical fields, which make it reasonable to foresee that 
there will soon be a convergence of materials chemistry, biology, and medicine towards the development of sophisticated materials and optoelectronic devices spontaneously assembled inside living organisms.

\section{References}

(1) (a) Archna; Pathania, S.; Chawla, P. A. Bioorg. Chem. 2020, 101, 104026. (b) Shah, R.; Verma, P. K. Chem. Cent. J. 2018, 12, 137. (c) Singh, A.; Singh, G.; Singh Bedi, P. M. J. Heterocycl. Chem. 2020, 57, 2658. (d) Apetrei, R. M.; Camurlu, P. J. Electrochem. Soc. 2020, 167, 037557. (e) Bhilare, N. V.; Auti, P. B.; Marulkar, V.S.; Pise, V.J. Mini-Rev. Med. Chem. 2021, 21, 217. (f) Amna, B.; Siddiqi, H. M.; Hassana, A.; Ozturk, T. RSC Adv. 2020, 10, 4322. (g) Li, L.; Zhao, C.; Wang, H. Chem. Rec. 2016, 16, 797. (h) Qiao, Y.; Yin, X.; Tang, C. Sci. China Chem. 2015, 58, 1641. (i) Perepichka, I. F.; Perepichka, D. F. Handbook of Thiophene-Based Materials: Applications in Organic Electronics and Photonics. Wiley: Chichester, 2009. (j) Rasmussen, S. C.; Evenson, S. J.; McCausland, C. B. Chem. Commun. 2015, 51, 4528. (k) Barbarella, G.; Zangoli, M.; Di Maria, F. Adv. Heterocycl. Chem. 2017, 123, 105. (l) Zangoli, M.; Di Maria, F.; Barbarella, G. ChemistryOpen 2020, 9, 499.

(2) Barbarella, G.; Zambianchi, M.; Bongini, A.; Antolini, L. Adv. Mater. 1993, 5, 384.

(3) Bongini, A.; Bottoni, A. J. Phys. Chem. A 1999, 103, 6800.

(4) Loi, M. A.; Da Como, E.; Dinelli, F.; Murgia, M.; Zamboni, R.; Biscarini, F.; Muccini, M. Nat. Mater. 2005, 4, 81.

(5) Melucci, M.; Barbarella, G.; Zambianchi, M.; Di Pietro, P.; Bongini, A. J. Org. Chem. 2004, 69, 4821.

(6) Melucci, M.; Gazzano, M.; Barbarella, G.; Cavallini, M.; Biscarini, F.; Maccagnani, P.; Ostoja, P. J. Am. Chem. Soc. 2003, 125, 10266.

(7) Milanese, E.; Brink, T.; Aghababaei, R.; Molinari, J. F. Nat. Commun. 2019, 10, 1116.

(8) Palamà, I. E.; Di Maria, F.; D’Amone, S.; Barbarella, G.; Gigli, G. J. Mater. Chem. B 2015, 3, 151.

(9) (a) Palamà, I.; Di Maria, F.; Viola, I.; Fabiano, E.; Gigli, G.; Bettini, C.; Barbarella, G. J. Am. Chem. Soc. 2011, 133, 17777. (b) Viola, I.; Palamà, I. E.; Coluccia, A. M. L.; Biasiucci, M.; Dozza, B.; Lucarelli, E.; Di Maria, F.; Barbarella, G.; Gigli, G. Integr. Biol. 2013, 5, 1057. (c) Moros, M.; Di Maria, F.; Dardano, P.; Tommasini, G.; CastilloMichel, H.; Kovtun, A.; Zangoli, M.; Blasio, M.; De Stefano, L.; Tino, A.; Barbarella, G.; Tortiglione, C. iScience 2020, 23, 101022.

(10) Chernyatina, A. A.; Nicolet, S.; Aebi, U.; Herrmann, H. Proc. Natl. Acad. Sci. U.S.A. 2012, 109, 13620.

(11) Melucci, M.; Barbarella, G.; Sotgiu, G. J. Org. Chem. 2002, 67, 8877.

(12) (a) Alesi, S.; Di Maria, F.; Melucci, M.; Macquarrie, D. J.; Luque, R.; Barbarella, G. Green Chem. 2008, 10, 517. (b) Di Maria, F.; Barbarella, G. J. Sulfur Chem. 2013, 34, 627.

(13) Mazzeo, M.; Vitale, V.; Della Sala, F.; Anni, M.; Barbarella, G.; Favaretto, L.; Sotgiu, G.; Cingolani, R.; Gigli, G. Adv. Mater. 2005, 17,34 .

(14) (a) Helten, H. Chem. Asian J. 2019, 14, 919. (b) Yin, X.; Liu, J.; Jäkle, F. Chem. Eur. J. 2021, 27, 2973.

(15) (a) Li, B.; Li, Z.; Guo, F.; Song, J.; Jiang, X.; Wang, Y.; Gao, S.; Wang, J.; Pang, X.; Zhao, L.; Zhang, Y. ACS Appl. Mater. Interfaces 2020, 12, 14233. (b) Li, D.; Hu, W.; Wang, J.; Zhang, Q.; Cao, X. M.; Ma, X.; Tian, H. Chem. Sci. 2018, 9, 5709.

(16) Cicoira, F.; Santato, C.; Melucci, M.; Favaretto, L.; Gazzano, M.; Muccini, M.; Barbarella, G. Adv. Mater. 2006, 18, 169.
(17) Chaudhry, M. U.; Muhieddine, K.; Wawrzinek, R.; Sobus, J.; Tandy, K.; Lo, S. C.; Namdas, E. B. Adv. Funct. Mater. 2020, 30 , 1905282.

(18) Viola, I.; Mazzeo, M.; Passabì, A.; D’Amone, S.; Cingolani, R.; Gigli, G. Adv. Mater. 2005, 17, 2935.

(19) (a) Viola, I.; Della Sala, F.; Piacenza, M.; Favaretto, L.; Gazzano, M.; Anni, M.; Barbarella, G.; Cingolani, R.; Gigli, G. Adv. Mater. 2007, 19, 1597. (b) Viola, I.; Piliego, C.; Favaretto, L.; Barbarella, G.; Cingolani, R.; Gigli, G. ACS Appl. Mater. Interfaces 2010, 2, 484.

(20) (a) Pisignano, D.; Anni, M.; Gigli, G.; Cingolani, R.; Zavelani-Rossi, M.; Lanzani, G.; Barbarella, G.; Favaretto, L. Appl. Phys. Lett. 2002, 81, 3534. (b) Pisignano, D.; Mele, E.; Persano, L.; Gigli, G.; Visconti, P.; Cingolani, R.; Barbarella, G.; Favaretto, L. Phys. Rev. B: Condens. Matter 2004, 70, 205206.

(21) Ghofraniha, N.; Viola, I.; Di Maria, F.; Barbarella, G.; Gigli, G.; Conti, C. Laser Photonics Rev. 2013, 7, 432.

(22) Sapienza, R. Nat. Rev. Phys. 2019, 1, 690.

(23) (a) Barbarella, G.; Favaretto, L.; Sotgiu, G.; Zambianchi, M.; Antolini, L.; Pudova, O.; Bongini, A. J. Org. Chem. 1998, 63, 5497. (b) Di Maria, F.; Zangoli, M.; Palamà, I. E.; Fabiano, E.; Zanelli, A.; Monari, M.; Perinot, A.; Caironi, M.; Maiorano, V.; Maggiore, A.; Pugliese, M.; Salatelli, E.; Gigli, G.; Viola, I.; Barbarella, G. Adv. Funct. Mater. 2016, 26, 6970.

(24) Rozen, S.; Bareket, Y. J. Org. Chem. 1997, 62, 1457.

(25) (a) Barbarella, G.; Favaretto, L.; Zambianchi, M.; Pudova, O.; Arbizzani, C.; Bongini, A.; Mastragostino, M. Adv. Mater. 1998, 10, 551. (b) Wei, S.; Xia, J.; Dell, E. J.; Jiang, Y.; Song, R.; Lee, H.; Rodenbough, P.; Briseno, A. L.; Campos, L. M. Angew. Chem. 2014, 126, 1863.

(26) Barbarella, G.; Favaretto, L.; Sotgiu, G.; Zambianchi, M.; Bongini, A.; Arbizzani, C.; Mastragostino, M.; Anni, M.; Gigli, G.; Cingolani, R. J. Am. Chem. Soc. 2000, 122, 11971.

(27) Li, J.; Wang, J.; Li, H.; Song, N.; Wang, D.; Tang, B. Z. Chem. Soc. Rev. 2020, 49, 1144.

(28) Antolini, L.; Tedesco, E.; Barbarella, G.; Favaretto, L.; Sotgiu, G.; Zambianchi, M.; Casarini, D.; Gigli, G.; Cingolani, R. J. Am. Chem. Soc. 2000, 122, 9006.

(29) Anni, M.; Della Sala, F.; Raganato, M. F.; Fabiano, E.; Lattante, S.; Cingolani, R.; Gigli, G.; Barbarella, G.; Favaretto, L.; Görling, A. J. Phys. Chem. B 2005, 109, 6004.

(30) Giglia, G.; Inganäs, O.; Anni, M.; De Vittorio, M.; Cingolani, R.; Barbarella, G.; Favaretto, L. Appl. Phys. Lett. 2001, 78, 1493.

(31) (a) Mariano, F.; Mazzeo, M.; Duan, Y.; Barbarella, G.; Favaretto, L.; Carallo, S.; Cingolani, R.; Gigli, G. Appl. Phys. Lett. 2009, 94, 063510. (b) Gigli, G.; Anni, M.; Theander, M.; Cingolani, R.; Barbarella, G. Synth. Met. 2001, 119, 581.

(32) Walzer, K.; Maennig, B.; Pfeiffer, M.; Leo, K. Chem. Rev. 2007, 107, 1233.

(33) (a) Dell, E. J.; Capozzi, B.; Xia, J.; Venkataraman, L.; Campos, L. M. Nat. Chem. 2015, 7, 209. (b) Busby, E.; Xia, J.; Low, J.Z.; Wu, Q.; Hoy, J.; Campos, L. M.; Sfeir, M. Y. J. Phys. Chem. B 2015, 119, 7644.

(34) (a) Wang, Y.; Feng, L.; Wang, S. Adv. Funct. Mater. 2019, 29, 1806818. (b) MacFarlane, L. R.; Shaikh, H.; Garcia-Hernandez, J. D.; Vespa, M.; Fukui, T.; Manners, I. Nat. Rev. Mater. 2021, 6, 7. (c) Zangoli, M.; Di Maria, F. View 2020, 2, 20200086. (d) Monti, F.; Manfredi, G.; Palamà, I. E.; Kovtun, A.; Zangoli, M.; D’Amone, S.; Ortolani, L.; Bondelli, G.; Szreder, T.; Bobrowski, K.; D’Angelantonio, M.; Lanzani, G.; Di Maria, F. Adv. Healthcare Mater. 2021, 10, 2001306. (e) Di Maria, F.; Lodola, F.; Zucchetti, E.; Benfenati, F.; Lanzani, G. Chem. Soc. Rev. 2018, 47, 4757. (f) Zangoli, M.; Di Maria, F.; Zucchetti, E.; Bossio, C.; Antognazza, M. R.; Lanzani, G.; 
Mazzaro, R.; Corticelli, F.; Baroncini, M.; Barbarella, G. Nanoscale 2017, 9, 9202. (g) Palamà, I. E.; Di Maria, F.; Zangoli, M.; D’Amone, S.; Manfredi, G.; Barsotti, J.; Lanzani, G.; Ortolani, L.; Salatelli, E.; Gigli, G.; Barbarella, G. RSC Adv. 2019, 9, 23036.

(35) (a) Maya-Vetencourt, J. F.; Manfredi, G.; Mete, M.; Colombo, E.; Bramini, M.; Di Marco, S.; Shmal, D.; Mantero, G.; Dipalo, M.; Rocchi, A.; DiFrancesco, M. L.; Papaleo, E. D.; Russo, A.; Barsotti, J.; Eleftheriou, C.; Di Maria, F.; Cossu, V.; Piazza, F.; Emionite, L.; Ticconi, F.; Marini, C.; Sambuceti, G.; Pertile, G.; Lanzani, G.; Benfenati, F. Nat. Nanotechnol. 2020, 15, 698. (b) Tortiglione, C.; Antognazza, M. R.; Tino, A.; Bossio, C.; Marchesano, V.; Bauduin, A.; Zangoli, M.; Morata, S. V.; Lanzani, G. Sci. Adv. 2017, 3, e1601699. (c) Bossio, C.; Aziz, I. A.; Tullii, G.; Zucchetti, E.; Debellis, D.; Zangoli, M.; Di Maria, F.; Lanzani, G.; Antognazza, M. R. Front. Bioeng. Biotechnol. 2018, 6, 1.

(36) Millstone, J. E.; Kavulak, D. F. J.; Woo, C. H.; Holcombe, T. W.; Westling, E. J.; Briseno, A. L.; Toney, M. F.; Fréchet, J. M. J. Langmuir 2010, 26, 13056.

(37) Di Maria, F.; Zanelli, A.; Liscio, A.; Kovtun, A.; Salatelli, E.; Mazzaro, R.; Morandi, V.; Bergamini, G.; Shaffer, A.; Rozen, S. ACS Nano 2017, 11, 1991.

(38) (a) Shimizu, H.; Yamada, M.; Wada, R.; Okabe, M. Polym. J. 2008, 40, 33. (b) Rao, J. P.; Geckeler, K. E. Prog. Polym. Sci. 2011, 36, 887.

(39) Melitz, W.; Shen, J.; Kummel, A. C.; Lee, S. Surf. Sci. Rep. 2011, 66, 1.

(40) Li, X.; Perera, K.; He, J.; Gumyusenge, A.; Mei, J. J. Mater. Chem. C 2019, 7, 12761.
(41) Österholm, A. M.; Shen, D. E.; Gottfried, D. S.; Reynolds, J. R. Adv. Mater. Technol. 2016, 1, 1600063.

(42) (a) Kim, Y.; Kim, Y.; Kim, S.; Kim, E. ACS Nano 2010, 4, 5277. (b) Moreira, T.; Maia, M.; Parola, A. J.; Zangoli, M.; Di Maria, F.; Laia, C. A. T. In Chemical Solution Synthesis for Materials Design and Thin Film Device Applications. Das, S.; Dhara, S. Elsevier: Amsterdam, 2021, 407.

(43) Moreira, T.; Laia, C. A. T.; Zangoli, M.; Antunes, M.; Di Maria, F.; De Monte, S.; Liscio, F.; Parola, A. J.; Barbarella, G. ACS Appl. Polym. Mater. 2020, 2, 3301.

(44) Zucchetti, E.; Zangoli, M.; Bargigia, I.; Bossio, C.; Di Maria, F.; Barbarella, G.; D’Andrea, C.; Lanzani, G.; Antognazza, M. R. J. Mater. Chem. B 2017, 5, 565.

(45) Barbarella, G.; Bongini, A.; Zambianchi, M. Macromolecules 1994, 27, 3039.

(46) Lehn, J. M. Angew. Chem. Int. Ed. 2015, 54, 3276.

(47) (a) Di Maria, F.; Zangoli, M.; Gazzano, M.; Fabiano, E.; Gentili, D.; Zanelli, A.; Fermi, A.; Bergamini, G.; Bonifazi, D.; Perinot, A.; Caironi, M.; Mazzaro, R.; Morandi, V.; Gigli, G.; Liscio, A.; Barbarella, G. Adv. Funct. Mater. 2018, 28, 1801946. (b) Zangoli, M.; Gazzano, M.; Monti, F.; Maini, L.; Gentili, D.; Liscio, A.; Zanelli, A.; Salatelli, E.; Gigli, G.; Baroncini, M.; Di Maria, F. ACS Appl. Mater. Interfaces 2019, 11, 16864. (c) Di Maria, F.; Fabiano, E.; Gentili, D.; Biasiucci, M.; Salzillo, T.; Bergamini, G.; Gazzano, M.; Zanelli, A.; Brillante, A.; Cavallini, M.; Della Sala, F.; Gigli, G.; Barbarella, G. Adv. Funct. Mater. 2014, 24, 4943. 